\title{
Spectroscopy of ground and excited states of pseudoscalar and vector charmonium and bottomonium
}

\author{
Hluf Negash and Shashank Bhatnagar
}

October 17, 2016

Department of Physics, Addis Ababa University, P.O.Box 1176, Addis Ababa, Ethiopia

\section{Abstract}

In this work we calculate the mass spectrum, weak decay constants, two photon decay widths, and two gluon decay widths of ground (1S), and radially excited $(2 \mathrm{~S}, 3 \mathrm{~S}, \ldots)$ states of pseudoscalar charmoniuum and bottomonium such as $\eta_{c}$ and $\eta_{b}$, as well as the mass spectrum and leptonic decay constants of ground $m$ state $(1 \mathrm{~S})$, excited $(2 \mathrm{~S}, 1 \mathrm{D}, 3 \mathrm{~S}, 2 \mathrm{D}, 4 \mathrm{~S}, \ldots, 5 \mathrm{D})$ states of vector charmonium and bottomonium such as $J / \psi$,

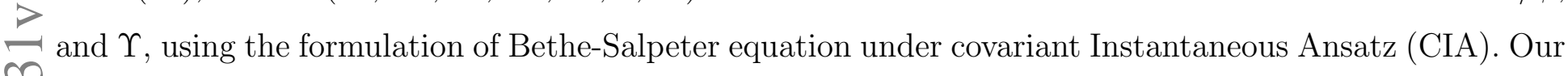
6 results are in good agreement with data (where ever available) and other models. In this framework, from . the beginning, we employ a $4 \times 4$ representation for two-body $(q \bar{q})$ BS amplitude for calculating both the $\bigcup_{n}^{\infty}$ mass spectra as well as the transition amplitudes. However, the price we have to pay is to solve a coupled ـ. set of equations for both pseudoscalar and vector quarkonia, which we have explicitly shown get decoupled in the heavy-quark approximation, leading to mass spectral equation with analytical solutions for both 궁 masses, as well as eigenfunctions for all the above states, in an approximate harmonic oscillator basis. The analytical forms of eigen functions for ground and excited states so obtained are used to evaluate the decay constants and decay widths for different processes.

\section{Introduction}

Heavy flavor mesons are of the type $Q \bar{Q}$, or $Q \bar{q}$, in which one of the quarks belongs to a heavy flavour such as $c$ or $b$. Quarkonia usually refers to heavy mesons with quark composition $(Q \bar{Q})$. Charmonium and bottomonium are two types of quarkonia that are observed with quark composition $c \bar{c}$ and $b \bar{b}$ respectively. Physically, the charmonium system is analogous to positronium system. However, historically, its role as 
a model system in QCD is similar in importance to the role of Hydrogen atom in Quantum mechanics, due to the fact that this system is non-relativistic and has a hydrogen atom like spectrum. Charmonium in fact occupies a valuable intermediate position within QCD, being neither in the purely non-relativistic regime nor the regime where chiral symmetry breaking dominates the physics. This makes it a relatively clean system in which to study non-perturbative QCD dynamics, QCD-inspired quark-potential models as well as lattice QCD, which have been rather successful in describing the observed features of the spectrum (see [1] for a review). However, charmonium cannot be considered to be completely understood; as an example, in recent years a number of new charmonium resonances have been claimed in experiment, several of which cannot be easily reconciled with the predictions of simple quark-potential models. Charmonium spectrum provided a simple example of how QCD works, which was made even more compelling with the subsequent observation of bottomonium spectra. Investigation of properties of these mesons gives a lot of information about heavy quark dynamics. Below the heavy flavour meson pair $(D \bar{D})$ production threshold, all charmonia states have been well established, and there is a good agreement between predictions based on potential models and data. However, there are many charmonium and charmonium like states [2, 3, 4, 4, 5] observed above $D \bar{D}$ threshold in the past ten years. Some of these are good candidates for the charmonia predicted in different models. However, many have unusual quantum numbers [2, 3, 4, 5] (which can not be reached by pure quark states), indicating that exotic states such as multi - quark states, molecule, hybrid, or glueball may have been observed. The spectroscopy and decay rates of quarkonia are quite important to study as huge amount of high precession data acquired from many experimental facilities world over are continuously providing accurate information about hadrons particularly in charm and beauty flavour sectors. The mass spectrum and the decays of these bound states can be tested experimentally and thus studies on them may throw valuable insight of the heavy quark dynamics and lead to a deeper understanding of QCD further.

As regards the dynamical framework, to investigate these properties is concerned, many non-perturbative approaches, such as Lattice QCD [6, 7, 8], Chiral perturbation theory [9], heavy quark effective theory [10], QCD sum rules [11, 12], N.R.QCD [13], dynamical-equation based approaches like Schwinger-Dyson equation and Bethe-Salpeter equation (BSE) [14, 15, 16, 17, 18, 19, 20], and potential models [21, 22] have been proposed to deal with the long distance property of QCD. Some of the interesting works on heavy-quark spectroscopy in the framework of BSE are [23, 24].

Many theoretical predictions on the decay properties particularly the leptonic and two-gamma decays of quarkonia based on the relativistic quark model or potential model [25, 26, 27], Bethe-Salpeter equation [28, 29], heavy-quark spin symmetry [30] and lattice QCD [31] are available in literature. Such studies have become a hot topic in recent years, due to observation of many new states at various high 
energy accelerators at BABAR, Belle, CLEO and BES-III collaborations [32, 33, 34, 35]. All this has opened up new challenges in theoretical understanding of heavy hadrons and provide an important tool for exploring the structure of these simplest bound states in QCD and for studying the non-perturbative (long distance) behavior of strong interactions.

We wish to mention that though heavy quarkonia $(c \bar{c}$ and $b \bar{b})$ are very well described in terms of NR potential models, and relativized potential models (like [22]) can treat light mesons simultaneously with heavy mesons, however, they cannot be regarded as an ideal theory that is closest to QCD, due to their inability in implementing Lorentz and gauge invariance for a consistent treatment of motion of quarks within the hadron. Most of such models, employ the $v^{2} / c^{2}$ corrections, or the replacement $\frac{p^{2}}{2 m} \rightarrow\left(p^{2}+m^{2}\right)^{1 / 2}$ in the kinetic energy operator (for each quark) in a Schrodinger type equation, which provides the bulk of momentum dependent effects. However, interaction terms require a more substantial form of treatment consistent with relativity. We further wish to point out that though, in heavy quarkonia, NR treatment may at best be a good first approximation, but in charmonium (where charmed quark is not heavy enough to be considered non-relativistically), one finds typical velocities, $\frac{v}{c} \sim 0.4$, making relativistic effects in charmonium important, which is specially so for electroweak decay properties as well as various other transitions at high energies, as mentioned above. These decay widths are sensitive to relativistic effects (see [16]). Thus NR and semi-relativistic equations (as in [22]) are not completely adequate as proper starting point for light, and semi-light quark systems.

In this respect, the Bethe-Salpeter Equation (BSE), with its 4D Lorentz-invariant structure is a natural candidate for hadron physics, since it is firmly rooted in field theory, and its general framework rests on Lorentz and gauge invariance, due to which it has a very wide applicability, all the way from low energy spectroscopy to high energy transition amplitudes, and can be used to study both light and heavy quark systems in an integrated manner. Thus the BSE framework provides a realistic description for analyzing hadrons as composite objects. Despite its drawback of having to input model-dependent kernel, these studies have become an interesting topic in recent years, since calculations have shown that BSE framework using phenomenological potentials can give satisfactory results on more and more data are being accumulated. The Lorentz-invariance at the input form of BSE formally ensures a continued validity of predictive powers from low energy spectroscopy to high energy processes within a common dynamical framework. The 4D BS wave function [15, 36, 37, 38, 39, 40] (that carries the entire non-perturbative information) arises as solution of the 4D BS equation. Since this wave function is determined as a solution of the formal dynamical equation, it does not suffer from the uncertainties of a variational determination (as in NR approaches [22]), and is able to carry over the micro causality information contained in the BSE. From non-perturbative 4D BS wave function, one can work out the 4D hadron-quark vertices, that are 
effective coupling vertices of hadron with all its constituents (quarks), and then naturally work out transition amplitudes for different processes over a vast range of energies, employing Feynman diagrammatic techniques over various quark-loop diagrams corresponding to the process considered.

A number of recent studies in the framework of BSE have been carried out on leptonic decays and two photon decays of mesons. The decay into two photons is considered as an interesting experimental playground in the mesonic physics of the near future. The two-photon decay of mesons can be used to identify the flavor of quark and anti-quark states. Two photon couplings also provide a useful probe of the internal structure of mesons. The states such as, $\eta_{c}$ and $\eta_{b}$ have been observed to have partial widths consistent with quark model predictions. These states can also decay into two gluons [41], which accounts for a substantial portion of the hadronic decays for states below the $c \bar{c}$ or $b \bar{b}$ threshold. It is clearly important to have accurate quark model predictions of widths for all experimentally accessible $q \bar{q}$-mesons. Thus, in this work we are predominantly interested in studying the mass spectrum and leptonic decays of ground and excited states of pseudoscalar and vector quarkonia, such as $\eta_{c}, \eta_{b}, J / \psi$, and $\Upsilon$ respectively (which proceed through the coupling of quark- anti-quark loop to the axial vector and vector currents respectively), as well as, the two-photon radiative decays, and two-gluon decays of ground state $(1 S)$ and radial excitations $(2 S$ and $3 S)$ of conventional pseudoscalar mesons such as $\eta_{c}$ and $\eta_{b}$ in the framework of BSE under Covariant Instantaneous Ansatz (CIA) [15, 20, 37, 38, 39, 40], though some of the excited states have not yet been experimentally discovered.

We have employed the BSE framework under Covariant Instantaneous Ansatz (CIA). CIA is a Lorentzinvariant generalization of the instantaneous ansatz (IA). What distinguishes CIA from other threedimensional (3D) reductions of BSE is its capacity for a two-way interconnection [15, 20, 37, 40]: an exact 3D BSE reduction for a $q \bar{q}$ system (for calculation of the mass spectrum), and an equally exact reconstruction of original 4D BSE (for calculation of transition amplitudes as 4D quark loop integrals). In some of our previous works in BSE under CIA, we had made use of a confinement potential $V\left(\widehat{q}, \widehat{q}^{\prime}\right)$ given in Eq.(33), for a number of successful predictions for not only the mass spectrum [15, 36, 40] of light $(q \bar{q})$ and some heavy mesons, but also a number of processes such as leptonic decays of vector mesons such as: $\rho, \omega, \phi, J / \psi$, and $\Upsilon$ [40] through the process $V \rightarrow e^{+}+e^{-}$, leptonic decays of pseudoscalar mesons such as $\pi, K, D, D_{S}$ and $B$ through the process, $P \rightarrow l+\overline{\nu_{\bar{l}}}$ and two-photon decays of $\pi$, and $\eta_{c}$ mesons [39] through process, $P \rightarrow \gamma+\gamma$, as well as the processes proceeding through quark triangle loop diagrams involving two or more hadron-quark vertices, such as: single photon decays of $\rho^{0, \pm}$, and $\omega^{0}$ mesons through process $V \rightarrow P+\gamma$, as well as strong decays of first radial excitations of lightest vector meson states $\rho(1450)$, and $\omega(1420)$ [42] through process $V^{\prime} \rightarrow V+P$ (where $V$, and $P$ refer to vector and pseudoscalar mesons). 
However this form of confining potential was employed mostly for light mesons, and for some cases to heavy mesons. However, in our previous BSE framework [38, 39, 40], we had adopted a $16 \times 1$ column representation for two-body $(q \bar{q}$ or $q q)$ BS amplitude (though both $16 \times 1$ and $4 \times 4$ representations of BSE are completely equivalent [14]). We had employed Gordon reduction on the mass shells of individual quarks to obtain the 3D mass spectral equation. And for bringing out the structure of the full 4D BS wave function in a $4 \times 4$ matrix form (that is needed for calculation of transition amplitudes through quark-loop diagrams), we made use of standard transformations [14] of charge conjugation of spinors. This is in contrast to the BSE framework employed in this paper, where we have employed a $4 \times 4$ framework of BSE from the very beginning (with the same confining potential in Eq.(33)), and used it for both the mass spectral predictions as well as the transition amplitude calculations involving heavy quarkonia (both pseudoscalar and vector), though the price one has to pay for this approach, is to solve a set of coupled equations, to obtain the masss spectral equations. However, in the present work, we have explicitly shown that in the heavy quark approximation (valid for $c \bar{c}$, and $b \bar{b}$ systems), these equations can be decoupled, and analytical solutions (both mass spectrum and eigen functions) of these equations can be obtained using approximate harmonic oscillator basis.

\begin{tabular}{ccccccccccc}
\hline$L_{J}^{2 S+1}$ & $S_{0}^{1}$ & $S_{1}^{3}$ & $P_{1}^{1}$ & $P_{0}^{3}$ & $P_{1}^{3}$ & $P_{2}^{3}$ & $D_{2}^{1}$ & $D_{1}^{3}$ & $D_{2}^{3}$ & $D_{3}^{3}$ \\
\hline$J^{P C}$ & $0^{-+}$ & $1^{--}$ & $1^{+-}$ & $0^{++}$ & $1^{++}$ & $2^{++}$ & $2^{-+}$ & $1^{--}$ & $2^{--}$ & $3^{--}$ \\
\hline
\end{tabular}

Table 1: Quantum numbers of lowest quarkonium states. These quantum numbers are repeated for the radial excitations of these states

Towards this end, we first analyze the quarkonium states according to their total angular momentum, $\vec{J}=\vec{L}+\vec{S}$, parity $P=(-1)^{L+1}$, and charge conjugation, $C=(-1)^{L+S}$ for states classified as $J^{P C}$, with the lowest possible states for quarkonia are listed in Table 1. We start with the most general structure of Bethe-Salpeter wave function for $J^{P C}=0^{-+}$and $J^{P C}=1^{--}$. It can be seen from Table 1 , that $1^{--}$ state has not only the ground state $(1 S)$ components, but also the orbital $(1 D)$ excitations. The same holds true for their radial excitations. We have started with the full Dirac structure of these states with all the Dirac covariants multiplying various scalar functions of internal hadron momentum, $q$ as in [14], with various Dirac structures incorporated into the wave functions in accordance with the power counting rule [38, 40] suggested recently. We then put the formulated BS wave functions into the instantaneous BSE and turn the equation into a set of proper coupled equations [43] for the components which appear in the formulation. These equations are then explicitly shown to decouple in the heavy-quark limit, and are reduced to a single mass spectral equation, whose analytic solutions in an approximate harmonic 
oscillator basis yield the mass spectrum and wave functions for ground and excited states of $\eta_{c}, \eta_{b}, J / \psi$ and $\Upsilon$. For this we need 6 input parameters (that include two input quarks masses $m_{c}$, and $m_{b}$ ). We then derive the leptonic decay constants, and the decay widths for two-photon decays, and two-gluon decays of pseudoscalar quarkonia $\eta_{c}$, and $\eta_{b}$ for their ground and radially excited states, as well as the leptonic decay constants for ground, radially and orbitally excited states of vector quarkonia, $J / \psi$, and $\Upsilon$, with the 6 input parameters fixed above.

This paper is structured as follows: in section $\mathbf{2}$ we introduce the BSE and formulate the instantaneous BSE for pseudoscalar and vector quarkonia. In section 3 we start with the generalized formulation of BS wave functions for pseudoscalar $\left(J^{P C}=0^{-+}\right)$and vector $\left(J^{P C}=1^{--}\right)$quarkonia, with definite quantum numbers and derive their mass spectral coupled equations. In section $\mathbf{4}$, we study the leptonic decays of pseudoscalar and vector quarkonia. In section $\mathbf{5}$, we derive the two photon and two gluon decays of pseudoscalar quarkonia. Finally, section 6 is relegated to numerical results and discussion.

\section{Formulation of BSE under CIA}

Lets consider a $q \bar{q}$ comprising of fermionic quarks of masses $m_{1}$ and $m_{2}$ respectively. We start with a $4 \mathrm{D}$ BSE for $q \bar{q}$ system, written in a $4 \times 4$ representation of $4 \mathrm{D} \operatorname{BS}$ wave function $\Psi(P, q)$ as:

$$
S_{F}^{-1}\left(p_{1}\right) \Psi(P, q) S_{F}^{-1}\left(-p_{2}\right)=\frac{i}{(2 \pi)^{4}} \int d^{4} q^{\prime} K\left(q, q^{\prime}\right) \Psi\left(P, q^{\prime}\right)
$$

where $K\left(q, q^{\prime}\right)$ is the interaction kernel between the quark and anti-quark, and $p_{1,2}$ are the momenta of the quark and anti-quark, which are related to the internal 4-momentum $q$ and total momentum $P$ of hadron of mass $M$ as,

$$
p_{1,2 \mu}=\hat{m}_{1,2} P_{\mu} \pm q_{\mu}
$$

where $\hat{m}_{1,2}=\frac{1}{2}\left[1 \pm \frac{\left(m_{1}^{2}-m_{2}^{2}\right)}{M^{2}}\right]$ are the Wightman-Garding (WG) definitions of masses of individual quarks which ensure that on the mass shells $(P . q=0)$ of either quarks, even when $m_{1} \neq m_{2}$. However for equal mass mesons, $\left(m_{1}=m_{2}=m\right)$, we have $\hat{m}_{1}=\hat{m}_{2}=\frac{1}{2}$.

Then $p_{1,2 \mu}$ becomes,

$$
p_{1,2 \mu}=\frac{1}{2} P_{\mu} \pm q_{\mu}
$$

Now it is convenient to express the internal momentum of the hadron $q_{\mu}$ as the sum of two parts. They are: (i) the transverse component, $\hat{q}_{\mu}=q_{\mu}-(q \cdot P) P_{\mu} / P^{2}$ which is orthogonal to total hadron momentum $P_{\mu}$ (ie. $\hat{q} \cdot P=0$ regardless of whether the individual quarks are on-shell or off-shell), and 
(ii) the longitudinal component, $\sigma P_{\mu}=(q \cdot P) P_{\mu} / P^{2}$, which is parallel to $P_{\mu}$. Thus we can decompose $q_{\mu}$ as, $q_{\mu}=(M \sigma, \widehat{q})$, where the transverse component, $\widehat{q}$ is an effective 3D vector, while the longitudinal component, $M \sigma$ plays the role of the time component. The 4-D volume element in this decomposition is, $d^{4} q=d^{3} \hat{q} M d \sigma$. To obtain the 3D BSE and the hadron-quark vertex, use an Ansatz on the BS kernel $K$ in Eq. (1) which is assumed to depend on the $3 \mathrm{D}$ variables $\hat{q}_{\mu}, \hat{q}_{\mu}^{\prime}$ as,

$$
K\left(q, q^{\prime}\right)=K\left(\hat{q}, \hat{q}^{\prime}\right)
$$

Hence, the longitudinal component, $M \sigma$ of $q_{\mu}$, does not appear in the form $K\left(\hat{q}, \hat{q}^{\prime}\right)$ of the kernel. For reducing Eq.(1) to 3D form, we define $3 \mathrm{D}$ wave function $\psi(\hat{q})$ as:

$$
\psi(\hat{q})=\frac{i}{2 \pi} \int M d \sigma \Psi(P, q)
$$

Substituting Eq.(5) in eq.(1), with definition of kernel in eq.(4), we get a covariant version of Salpeter equation,

$$
\left(\not p_{1}-m_{1}\right) \Psi(P, q)\left(\not p_{2}+m_{2}\right)=\int \frac{d^{3} \hat{q}^{\prime}}{(2 \pi)^{3}} K\left(\hat{q}, \hat{q}^{\prime}\right) \psi\left(\hat{q}^{\prime}\right),
$$

and the $4 \mathrm{D}$ BS wave function becomes,

$$
\Psi(P, q)=S_{F}\left(p_{1}\right) \Gamma(\hat{q}) S_{F}\left(-p_{2}\right)
$$

where

$$
\Gamma(\hat{q})=\int \frac{d^{3} \hat{q}^{\prime}}{(2 \pi)^{3}} K\left(\hat{q}, \hat{q}^{\prime}\right) \psi\left(\hat{q}^{\prime}\right)
$$

plays the role of hadron-quark vertex function. The kernel is taken to have $\gamma_{\mu} \otimes \gamma^{\mu}$ form, details of which are given in the next section. Thus with this form of the kernel, we can write $\Gamma(\hat{q})=\int \frac{d^{3} \hat{q}^{\prime}}{(2 \pi)^{3}} V\left(\hat{q}, \hat{q}^{\prime}\right) \gamma_{\mu} \psi\left(\hat{q}^{\prime}\right) \gamma^{\mu}$ (where $V$ is spatial part of the kernel). We can to a good approximation express $\gamma_{\mu} \psi\left(\hat{q}^{\prime}\right) \gamma^{\mu} \approx \Theta \psi\left(\hat{q}^{\prime}\right)$, where $\Theta$ involves the spin-spin interactions alone, that factor out of the RHS of the hadron-quark vertex on taking the dominant Dirac structures in $\psi\left(\hat{q}^{\prime}\right)$ in the calculation of $\Theta$, and we can write $\Gamma(\hat{q})=$ $\Theta \int \frac{d^{3} \hat{q}^{\prime}}{(2 \pi)^{3}} V\left(\hat{q}, \hat{q}^{\prime}\right) \psi\left(\hat{q}^{\prime}\right)$. And, $S_{F}(p)$ is the usual fermionic propagator of the quarks, given as,

$$
S_{F}\left( \pm p_{1,2}\right)=\frac{\not p_{1,2} \pm m_{1,2}}{\Delta_{1,2}}
$$

where $\Delta_{1,2}=p_{1,2}^{2} \mp m_{1,2}^{2}$, which can also be decomposed as in[19] ,

$$
S_{F}\left( \pm p_{i}\right)=\frac{\Lambda_{i}^{+}(\hat{q})}{I(i) M \sigma+\frac{1}{2} M-\omega_{i}}+\frac{\Lambda_{i}^{-}(\hat{q})}{I(i) M \sigma+\frac{1}{2} M+\omega_{i}}
$$

with

$$
\begin{aligned}
& \omega_{i}^{2}=m_{i}^{2}+\hat{q}^{2} \\
& \Lambda_{i}^{ \pm}(\hat{q})=\frac{1}{2 \omega_{i}}\left[\frac{\not P}{M} \omega_{i} \pm I(i)\left(m_{i}+\not \hat{q}\right)\right]
\end{aligned}
$$


where $i=1,2$ for quark and anti-quark respectively, and $I(i)=(-1)^{i+1}$. Here $\Lambda_{i}^{ \pm}(\hat{q})$, are called as projection operators. With the projected wave functions, one can rewrite the BSE as,

$$
\left(\frac{1}{2} M \mp \omega_{1}+M \sigma\right)\left(\frac{1}{2} M \mp \omega_{2}-M \sigma\right) \Psi^{ \pm \pm}(P, q)=\Lambda_{1}^{ \pm}(\hat{q}) \Gamma(\hat{q}) \Lambda_{2}^{ \pm}(\hat{q})
$$

where projected wave functions, $\psi^{ \pm \pm}(\hat{q})$ are obtained by the operation of projection operators on $\psi(\widehat{q})$ as,

$$
\psi^{ \pm \pm}(\hat{q})=\Lambda_{1}^{ \pm}(\hat{q}) \frac{\not P}{M} \psi(\hat{q}) \frac{\not p}{M} \Lambda_{2}^{ \pm}(\hat{q})
$$

With contour integration over $d \sigma$ on both sides of Eq.(12), we can obtain:

$$
\psi(\hat{q})=-\frac{\Lambda_{1}^{+}(\hat{q}) \Gamma(\hat{q}) \Lambda_{2}^{+}(\hat{q})}{M-\omega_{1}-\omega_{2}}+\frac{\Lambda_{1}^{-}(\hat{q}) \Gamma(\hat{q}) \Lambda_{2}^{-}(\hat{q})}{M+\omega_{1}+\omega_{2}}
$$

The complete wave function can separate in four parts as:

$$
\psi(\hat{q})=\psi^{++}(\hat{q})+\psi^{+-}(\hat{q})+\psi^{-+}(\hat{q})+\psi^{--}(\hat{q})
$$

The BSE then reduces to four independent equations as [43]:

$$
\begin{aligned}
& (M-2 \omega) \psi^{++}(\hat{q})=-\Lambda_{1}^{+}(\hat{q}) \Gamma(\hat{q}) \Lambda_{2}^{+}(\hat{q}) \\
& (M+2 \omega) \psi^{--}(\hat{q})=\Lambda_{1}^{-}(\hat{q}) \Gamma(\hat{q}) \Lambda_{2}^{-}(\hat{q}) \\
& \psi^{+-}(\hat{q})=\psi^{-+}(\hat{q})=0
\end{aligned}
$$

where $\omega_{1}=\omega_{2}(=\omega)$, for equal mass systems. In fact the four equations constitute an eigenvalue problem that should lead to evaluation of mass spectra of pseudoscalar (for preliminary work, see [43]) and vector charmonium and bottomonium states such as $\eta_{c}, \eta_{b}, J / \psi$ and $\Upsilon$. The framework is quite general so far. Thus to obtain the mass spectral equation, we have to start with the above four equations to solve the instantaneous BS equation.

\section{Derivation of mass spectral equations of pseudoscalar and vector quarkonia}

In this section, we show the method to solve an Instantaneous BS equation, which is first applied to the calculation of the mass spectrum of equal mass heavy pseudoscalar and vector quarkonia. We first write down the most general formulation of the relativistic BS wave functions, according to the total angular momentum $(\mathrm{J})$, parity $(\mathrm{P})$ and charge conjugation $(\mathrm{C})$ of the concerned bound state. We then put this BS wave function in Eq.(16) to derive the mass spectral equations, which are a set of coupled equations. We now illustrate this procedure for pseudoscalar and vector quarkonia. 
- For pseudoscalar mesons, the complete decomposition of 4D BS wave function in terms of various Dirac structures and scalar functions $\phi_{j}(P, q)$ multiplying them is [14]:

$$
\Psi^{P}(P, q)=\left\{\phi_{1}(P, q)+\not P \phi_{2}(P, q)+\not 1 \phi_{3}(P, q)+[\not P, \not q] \phi_{4}(P, q)\right\} \gamma_{5}
$$

where $\phi_{j}=\phi_{j}\left(q^{2}, q . P, P^{2}\right) ;(j=1,2,3,4)$ are the Lorentz scalar amplitudes multiplying the various Dirac structures in the BS wave function, $\Psi(P, q)$. Now in our framework, by use of a naive power counting rule in [38, 39, 40], we had shown that the Dirac structures associated with amplitudes $\phi_{1}$ and $\phi_{2}$ are leading, while the structures associated with $\phi_{3}$ and $\phi_{4}$ are sub-leading, and would contribute lesser to meson observable calculations in comparison to leading Dirac structures associated with $\phi_{1}$ and $\phi_{2}$. And in various calculations [38, 39, 40], we had shown that among the two leading Dirac structures associated with amplitudes $\phi_{1}$, and $\phi_{2}$, the structure associated with $\phi_{1}$ (i.e. $\left.\gamma_{5}\right)$ is dominant. We wish to point out that Munczek and Jain [44] have also earlier shown that $\phi_{1}$ is the dominant amplitude for all ground state pseudoscalar mesons. V.Sauli [45] has recently shown that the same is valid for their excited states as well. And this is particularly true for mesons made from heavy flavour quarks.

In the center of mass frame, where $q_{\mu}=(0, \widehat{q})$, we can then write the general decomposition of the instantaneous BS wave function for pseudoscalar mesons $\left(J^{P C}=0^{-+}\right)$, of dimensionality $M$ in the center of mass frame as [18]:

$$
\psi^{P}(\hat{q}) \approx\left[M \phi_{1}(\hat{q})+\not P \phi_{2}(\hat{q})+\not 1 \phi_{3}(\hat{q})+\frac{\not P \not 1}{M} \phi_{4}(\hat{q})\right] \gamma_{5}
$$

where $\phi_{1}, \phi_{2}, \phi_{3}$ and $\phi_{4}$ are even functions of $\hat{q}$ and $\mathrm{M}$ is the mass of the bound state (of the corresponding meson). We now obtain the algebraic forms of these amplitudes.

For this, we put the instantaneous BS wave function $\psi^{P}(\widehat{q})$ (Eq.(18)) into the last two equations of Eq.(16), and this leads to independent constraints on the components for the Instantaneous BS wave function as in [18, 19, 24]:

$$
\phi_{3}=0 ; \phi_{4}=\frac{-\phi_{2} M}{m}
$$

So we can apply the obtained constraints Eq.(19) to Eq.(18) and rewrite the relativistic wave function of state $\left(0^{-+}\right)$as,

$$
\psi^{P}(\hat{q}) \approx\left[M \phi_{1}(\hat{q})+\not P \phi_{2}(\hat{q})+\frac{\not 1 P}{m} \phi_{2}(\hat{q})\right] \gamma_{5}
$$

One can then see that the Instantaneous BS wave function of $0^{-+}$state is determined by only two independent functions $\phi_{1}$, and $\phi_{2}$. Putting the wave function Eq.(20) into the first two equations of 
Eq.(16) and by evaluating trace over the $\gamma$-matrices on both sides, we obtain two BS coupled integral equations,

$$
\begin{aligned}
& (M-2 \omega)\left[\phi_{1}(\hat{q})+\frac{\omega}{m}\left(1-\frac{\hat{q}^{2}}{m^{2}}\right) \phi_{2}(\hat{q})\right]=\Theta_{P} \int \frac{d^{3} \hat{q}^{\prime}}{(2 \pi)^{3}} V\left(\hat{q}, \hat{q}^{\prime}\right)\left[\phi_{1}\left(\hat{q}^{\prime}\right)+\frac{\omega}{m}\left(1+\frac{\left.\hat{q} \cdot \hat{q}^{\prime}\right)}{m^{2}} \phi_{2}\left(\hat{q}^{\prime}\right)\right]\right. \\
& \left.(M+2 \omega)\left[\phi_{1}(\hat{q})-\frac{\omega}{m}\left(1-\frac{\hat{q}^{2}}{m^{3}}\right) \phi_{2}(\hat{q})\right]=-\Theta_{P} \int \frac{d^{3} \hat{q}^{\prime}}{(2 \pi)^{3}} V\left(\hat{q}, \hat{q}^{\prime}\right)\left[\phi_{1}\left(\hat{q}^{\prime}\right)-\frac{\omega}{m}\left(1+\frac{\hat{q} \cdot \hat{q}^{\prime}}{m^{3}}\right) \phi_{2}\left(\hat{q}^{\prime}\right)\right] 21\right)
\end{aligned}
$$

To decouple these equations, we first add them. Then we subtract the second equation from the first equation. For a kernel that can be expressed as $V\left(\widehat{q}-\widehat{q}^{\prime}\right)=\bar{V}(\widehat{q}) \delta^{3}\left(\widehat{q}-\widehat{q}^{\prime}\right)$, we get two algebraic equations which are still coupled. Then from one of the two equations so obtained, we eliminate $\phi_{1}(\hat{q})$ in terms of $\phi_{2}(\hat{q})$, and plug this expression for $\phi_{1}(\hat{q})$ in the second equation of the coupled set so obtained to get a decoupled equation in $\phi_{2}(\hat{q})$. Similarly, we eliminate $\phi_{2}(\hat{q})$ from the second equation of the set of coupled algebraic equations in terms of $\phi_{1}(\hat{q})$, and plug it into the first equation to get a decoupled equation entirely in $\phi_{1}(\hat{q})$. Thus, we get two identical decoupled equations, one entirely in $\phi_{1}(\hat{q})$, and the other that is entirely in, $\phi_{2}(\hat{q})$. Employing the limit, $\omega \approx m$ on RHS, these equations can be expressed as:

$$
\begin{aligned}
& {\left[\frac{M^{2}}{4}-m^{2}-\widehat{q}^{2}\right] \phi_{1}(\hat{q})=\Theta_{P} \bar{V}(\widehat{q}) m \phi_{1}(\hat{q})+\frac{\Theta_{P}^{2}}{4} \bar{V}^{2}(\widehat{q}) \phi_{1}(\hat{q})} \\
& {\left[\frac{M^{2}}{4}-m^{2}-\widehat{q}^{2}\right] \phi_{2}(\hat{q})=\Theta_{P} \bar{V}(\widehat{q}) m \phi_{2}(\hat{q})+\frac{\Theta_{P}^{2}}{4} \bar{V}^{2}(\widehat{q}) \phi_{2}(\hat{q}) .}
\end{aligned}
$$

It is to be mentioned that these two decoupled Eqs. (22) would resemble h.o. equations, but for $\bar{V}^{2}(\widehat{q})$ term on the RHS of these equations. It will be shown later from the definition of kernel in Eq.(31), and Eq.(34), that this second term involving $\bar{V}^{2}(\widehat{q})$ is negligible in comparison to the first term involving $\bar{V}(\widehat{q})$ on the RHS, and can be dropped, and these equations would then resemble exact harmonic oscillator equations. However, due to identical nature of these equations, their solutions are written as: $\phi_{1}(\hat{q})=\phi_{2}(\hat{q}) \approx \phi_{P}(\hat{q})$, which represent the eigenfunction of the pseudoscalar meson obtained by solving the full Salpeter equation and we can write the wave function for $\left(J^{P C}=0^{-+}\right)$ state as:

$$
\psi^{P}(\hat{q}) \approx\left[M+\not P+\frac{\not 1 P}{m}\right] \phi_{P}(\hat{q}) \gamma_{5}
$$

In view of the arguments made above, in the structure of $\psi^{P}(\hat{q})$ for pseudoscalar mesons, $M \gamma_{5}$ would have dominant contribution among all the other Dirac structures ( and this is more so for heavy $Q \bar{Q}$ mesons)

- For vector mesons, the complete decomposition of $4 \mathrm{D}$ BS wave function in terms of various Dirac structures is [14]: 


$$
\begin{aligned}
& \Psi^{V}(P, q)=\notin \chi_{1}+\notin \not P \chi_{2}+[q \cdot \epsilon-\notin q] \chi_{3}+[2 q . \epsilon \not P+\notin(\not P q-\not 1 P)] \chi_{4} \\
& (q . \epsilon) \chi_{5}+(q . \epsilon) \not P \chi_{6}+(q . \epsilon) q \chi_{7}+(q . \epsilon)(\not P \not q-\not 1 P) \chi_{8}
\end{aligned}
$$

where $\chi_{\alpha}=\chi_{\alpha}\left(q^{2}, q . P, P^{2}\right) ;(\alpha=1,2,3, \ldots, 8)$ are the Lorentz scalar amplitudes multiplying the various Dirac structures in the BS wave function, $\Psi(P, q)$. We again mention that in our framework, by use of a naive power counting rule in [38, 39, 40, we had shown that the Dirac structures associated with amplitudes $\chi_{1}$ and $\chi_{2}$ are of leading order. Those with amplitudes $\chi_{3}, \ldots, \chi_{6}$ are sub-leading. We had also shown that, the structures associated with $\chi_{7}$ and $\chi_{8}$ are more much more suppressed than even the sub-leading Dirac structures, and would contribute very little to meson observable (specially heavy mesons) calculations in comparison to Dirac structures associated with $\chi_{1}, \ldots, \chi_{6}$. Thus we ignore the Dirac structures associated with $\chi_{7}$ and $\chi_{8}$ (see [40] for details). And in various calculations [38, 40], we had shown that among the two leading Dirac structures associated with amplitudes $\chi_{1}$, and $\chi_{2}$, the structure associated with $\chi_{1}$ i.e. $\gamma . \epsilon$ is most dominant. In Ref. [44, 45], it was also shown that $\chi_{1}$ is the dominant amplitude for not only ground state vector mesons, but also for their higher excitations.

We thus write the instantaneous BS wave function $\psi^{V}(\hat{q})$ with dimensionality $M$ for vector quarkonia, $\left(J^{P C}=1^{--}\right)$up to sub-leading order (i.e. $O\left(1 / M^{1}\right)$ as per the power counting scheme [40]) as in case of pseudoscalar mesons, in the center of mass frame as:

$$
\begin{aligned}
& \psi^{V}(\hat{q})=M \notin \chi_{1}+\notin \not P \chi_{2}+[\notin \not 1-\hat{q} . \epsilon] \chi_{3}+[\not P \notin \not q-(\hat{q} . \epsilon) \not P] \frac{1}{M} \chi_{4}+(\hat{q} . \epsilon) \chi_{5} \\
& +(\hat{q} . \epsilon) \not P \frac{\chi_{6}}{M}
\end{aligned}
$$

where $\chi_{1}, \ldots, \chi_{6}$ are even functions of $\hat{q}$ and $M$ is the mass of the bound state (of the corresponding meson). We now derive the mass spectral coupled equations from Eq.(16). Putting Eq.(25) into the last two equations of Eq.(16), and we obtain the independent constraints on the components for the Instantaneous BS wave function:

$$
\begin{aligned}
& \chi_{5}=\frac{M \chi_{1}}{m} \\
& \chi_{4}=\frac{-\chi_{2} M}{m},
\end{aligned}
$$

with $\chi_{6}=\chi_{3}=0$. Applying the constraints in Eq.(26) to Eq.(26), we can rewrite the relativistic wave function of state $\left(J^{P C}=1^{--}\right)$as:

$$
\psi^{V}(\hat{q})=\left[M \notin+\hat{q} \cdot \epsilon \frac{M}{m}\right] \chi_{1}(\hat{q})+\left[\notin \not P+\frac{2 \not P \hat{q} \cdot \epsilon}{m}-\frac{\not P \notin \not q}{m}\right] \chi_{2}(\hat{q}),
$$


where, we have been able to express the instantaneous wave function $\psi^{V}(\widehat{q})$ in terms of only the leading Dirac structures associated with amplitudes $\chi_{1}$ and $\chi_{2}$. Putting the wave function Eq.(27) into the first two equations of Eq.(16) and by evaluating trace over the $\gamma$-matrices on both sides, we can obtain two independent BS coupled integral equations:

$$
\begin{aligned}
& (M-2 \omega)\left[\chi_{1}(\hat{q})-\frac{\omega}{m} \chi_{2}(\hat{q})\right]=\Theta_{V} \int \frac{d^{3} \hat{q}^{\prime}}{(2 \pi)^{3}} V\left(\hat{q}, \hat{q}^{\prime}\right)\left[\chi_{1}\left(\hat{q}^{\prime}\right)-\frac{\omega}{m} \chi_{2}\left(\hat{q}^{\prime}\right)\right] \\
& (M+2 \omega)\left[\chi_{1}(\hat{q})+\frac{\omega}{m} \chi_{2}(\hat{q})\right]=\Theta_{V} \int \frac{d^{3} \hat{q}^{\prime}}{(2 \pi)^{3}} V\left(\hat{q}, \hat{q}^{\prime}\right)\left[-\chi_{1}\left(\hat{q}^{\prime}\right)-\frac{\omega}{m} \chi_{2}\left(\hat{q}^{\prime}\right)\right]
\end{aligned}
$$

To decouple these equations, we proceed as in the above case of pseudoscalar mesons. We first add these above equations. Then subtract the second equation from the first. And for a kernel expressed as $V\left(\widehat{q}-\widehat{q}^{\prime}\right)=\bar{V}(\widehat{q}) \delta^{3}\left(\widehat{q}-\widehat{q}^{\prime}\right)$, we get two algebraic coupled equations in $\chi_{1}$ and $\chi_{2}$. We Eliminate $\chi_{1}$ from the first equation in terms of $\chi_{2}$, and plug it in the second equation to get an equation entirely in terms of $\chi_{2}$. Similarly, we eliminate $\chi_{2}$ from the second equation in terms of $\chi_{1}$, and plugging in the first equation to get an equation entirely in terms of $\chi_{1}$. We thus get two identical decoupled equations, one in $\chi_{1}(\hat{q})$, and the other in $\chi_{2}(\hat{q})$, and in the approximation, $\omega \approx m$, (valid for heavy quarkonia), these equations can be expressed as:

$$
\begin{aligned}
& {\left[\frac{M^{2}}{4}-m^{2}-\widehat{q}^{2}\right] \chi_{1}(\hat{q})=\Theta_{V} \bar{V}(\widehat{q}) m \chi_{1}(\hat{q})+\frac{\Theta_{V}^{2}}{4} \bar{V}^{2}(\widehat{q}) \chi_{1}(\hat{q})} \\
& {\left[\frac{M^{2}}{4}-m^{2}-\widehat{q}^{2}\right] \chi_{2}(\hat{q})=\Theta_{V} \bar{V}(\widehat{q}) m \chi_{2}(\hat{q})+\frac{\Theta_{V}^{2}}{4} \bar{V}^{2}(\widehat{q}) \chi_{2}(\hat{q}),}
\end{aligned}
$$

It is to be mentioned that these two decoupled equations would again resemble h.o. equations, but for $\bar{V}^{2}(\widehat{q})$, term on the RHS of these equations. It will be shown later from the definition of spatial part $\mathrm{V}$ of the kernel in Eq.(31), and Eq.(34), that this term is negligible in comparison to the first term involving $\bar{V}$ on the RHS, and can be dropped, and these equations would then resemble exact harmonic oscillator equations.

From the identical nature of both these equations, we can again express the solutions as, $\chi_{1}(\hat{q})=$ $\chi_{2}(\hat{q}) \approx \phi_{V}(\hat{q})$, and we can write the wave function for $\left(J^{P C}=1^{--}\right)$as:

$$
\psi^{V}(\hat{q}) \approx\left[M \notin+\hat{q} \cdot \epsilon \frac{M}{m}+\notin \not P+\frac{2 \not p \hat{q} \cdot \epsilon}{m}-\frac{\not P \notin \not \hat{q}}{m}\right] \phi_{V}(\hat{q}) .
$$

In the structure of $\psi^{V}(\hat{q})$ for vector meson, as argued above, $M \notin$ is the most dominant Dirac structure, among all the other Dirac structures above (and this is more so for heavy $Q \bar{Q}$ mesons). We wish to mention that the framework is quite general up to this point. We now introduce the BS kernel. 
Now, as regards the BS kernel $K\left(q, q^{\prime}\right)$ [15, 38, 39, 40] is concerned, it is taken to be one-gluon-exchange like as regards the spin dependence $\left(\gamma_{\mu} \otimes \gamma^{\mu}\right)$, and color dependence $\left(\frac{1}{2} \vec{\lambda}_{1} \cdot \frac{1}{2} \vec{\lambda}_{2}\right)$, and has a scalar part $V$ :

$$
\begin{aligned}
& K\left(q, q^{\prime}\right)=\left(\frac{1}{2} \vec{\lambda}_{1} \cdot \frac{1}{2} \vec{\lambda}_{2}\right)\left(\gamma_{\mu} \otimes \gamma^{\mu}\right) V\left(q-q^{\prime}\right) \\
& V\left(\hat{q}, \hat{q}^{\prime}\right)=\frac{3}{4} \omega_{q \bar{q}}^{2} \int d^{3} \vec{r}\left[r^{2}\left(1+4 \hat{m}_{1} \hat{m}_{2} A_{0} M_{>}^{2} r^{2}\right)^{-\frac{1}{2}}-\frac{C_{0}}{\omega_{0}^{2}}\right] e^{i\left(\hat{q}-\hat{q}^{\prime}\right) \cdot \vec{r}} \\
& \omega_{q \bar{q}}^{2}=4 M_{>} \hat{m}_{1} \hat{m}_{2} \omega_{0}^{2} \alpha_{s}\left(M_{>}^{2}\right) \\
& \alpha_{s}\left(M_{>}^{2}\right)=\frac{12 \pi}{33-2 n_{f}}\left[\log \left(\frac{M_{>}^{2}}{\wedge^{2}}\right)\right]^{-1} \\
& \hat{m}_{1,2}=\frac{1}{2}\left[1 \pm \frac{\left(m_{1}^{2}-m_{2}^{2}\right)}{M^{2}}\right] \\
& \kappa=\left(1+4 \hat{m}_{1} \hat{m}_{2} A_{0} M^{2} r^{2}\right)^{-\frac{1}{2}} \\
& M_{>}=\operatorname{Max}\left(M, m_{1}+m_{2}\right) .
\end{aligned}
$$

The scalar part is purely confining (as in Ref. [19, 23, 43], and the Martin potential [46] employed for heavy mesons). Here the proportionality of $\omega_{q \bar{q}}^{2}$ on $\alpha_{S}\left(Q^{2}\right)$ is needed to provide a more direct QCD motivation [15] to confinement, and $\omega_{0}^{2}$ is postulated as a spring constant which is common to all flavors. Here in the expression for $V\left(\widehat{q}, \widehat{q}^{\prime}\right)$, the constant term $C_{0} / \omega_{0}^{2}$ is designed to take account of the correct zero point energies, while the $A_{0}$ term $\left(A_{0}<<1\right)$ simulates an effect of an almost linear confinement for heavy quark sectors (large $m_{1}, m_{2}$ ), while retaining the harmonic form for light quark sectors (small $m_{1}, m_{2}$ ), as is believed to be true for QCD (see [15, 38, 40]). Hence the term $\left(1+4 \hat{m}_{1} \hat{m}_{2} A_{0} M^{2} r^{2}\right)^{-\frac{1}{2}}$ in the above expression is responsible for effecting a smooth transition from harmonic $(q \bar{q})$ to linear $(Q \bar{Q})$ confinement.

We now try to work on the spatial part $V\left(\widehat{q}, \widehat{q}^{\prime}\right)$ of the confining potential $K\left(\widehat{q}, \widehat{q}^{\prime}\right)$. If we take the parameter $A_{0}=0$ (which corresponds to case of light mesons $(q \bar{q})$, since due to $A_{0}<<0$, the square root factor in the denominator, $\left.\kappa=\left(1+4 \hat{m}_{1} \hat{m}_{2} A_{0} M^{2} r^{2}\right)^{-\frac{1}{2}}=1\right)$, and $V$ would look like:

$$
V^{\prime}\left(\hat{q}, \hat{q}^{\prime}\right)=\frac{3}{4} \omega_{q \bar{q}}^{2} \int d^{3} \vec{r}\left[r^{2}-\frac{C_{0}}{\omega_{0}^{2}}\right] e^{i\left(\hat{q}-\hat{q}^{\prime}\right) \cdot \vec{r}}
$$

(where $V^{\prime}$ is $V$ without the factor $\kappa$ in denominator). Making use of the fact that $-\vec{\nabla}_{\widehat{q}}^{2}$ is the fourier transform of $r^{2}$ in momentum space, and the colour factor for bound $q \bar{q}$ system, $\left(\frac{1}{2} \vec{\lambda}_{1} \cdot \frac{1}{2} \vec{\lambda}_{2}\right)=-\frac{4}{3}$, we can write

$$
V^{\prime}\left(\widehat{q}, \widehat{q}^{\prime}\right)=\omega_{q \bar{q}}^{2}(2 \pi)^{3}\left[\vec{\nabla}_{\widehat{q}}^{2}+\frac{C_{0}}{\omega_{0}^{2}}\right] \delta^{3}\left(\widehat{q}-\widehat{q}^{\prime}\right) .
$$

which can be written as, $V^{\prime}\left(\widehat{q}, \widehat{q}^{\prime}\right)=\overline{V^{\prime}}(\widehat{q}) \delta^{3}\left(\widehat{q}-\widehat{q}^{\prime}\right)$. Putting this spatial part in place of the spatial part of the kernel $V$ in Eq.(22) and Eq.(29) (with the second term on the RHS of each of these equations 
dropped for reasons explained next), these equations would resemble harmonic oscillator equations, and we can have analytical solutions for them. But for parameter $A_{0} \neq 0$ (i.e if we reintroduce the factor $\kappa$ ), we can write the complete potential $V\left(\widehat{q}, \widehat{q}^{\prime}\right)$ as:

$$
\begin{aligned}
& V\left(\hat{q}, \hat{q}^{\prime}\right)=\bar{V}(\hat{q}) \delta^{3}\left(\hat{q}-\hat{q}^{\prime}\right), \\
& \bar{V}(\hat{q})=\omega_{q \bar{q}}^{2}\left[\kappa \vec{\nabla}_{\hat{q}}^{2}+\frac{C_{0}}{\omega_{0}^{2}}\right](2 \pi)^{3}, \\
& \kappa=\left(1-A_{0} M^{2} \vec{\nabla}_{\hat{q}}^{2}\right)^{-1 / 2}
\end{aligned}
$$

As mentioned above, we have dropped the terms with $\bar{V}^{2}$, in comparison to the terms involving $\bar{V}$ on the RHS of Eqs. (22) and (29), as the coefficients, $\Omega_{P, V}^{\prime}=\frac{\Theta_{P, V}^{2}}{4} \omega_{q \bar{q}}^{4}$ associated with the former have a very small contribution $(\leq 0.638 \%)$ in comparison to the coefficient $\Omega_{P, V}=m \Theta_{P, V} \omega_{q \bar{q}}^{2}$ associated with the latter for both pseudoscalar and vector mesons, due to $\omega_{q \bar{q}}^{4}<<\omega_{q \bar{q}}^{2}$ for $\eta_{c}, \eta_{b}, J / \psi$ and $\Upsilon$. The numerical

\begin{tabular}{|c|c|c|c|c|c|c|}
\hline & $\Omega_{P}$ & $\Omega_{P}^{\prime}$ & $\frac{\Omega_{P}^{\prime}}{\Omega_{P}} \%$ & $\Omega_{V}$ & $\Omega_{V}^{\prime}$ & $\frac{\Omega_{V}^{\prime}}{\Omega_{V}} \%$ \\
\hline$\eta_{c}$ & 0.0558 & 0.000356 & $0.638 \%$ & & & \\
\hline$\eta_{b}$ & 0.4564 & 0.00202 & $0.4426 \%$ & & & \\
\hline$J / \psi$ & & & & 0.0246 & 0.00006889 & $0.280 \%$ \\
\hline$\Upsilon$ & & & & 0.2293 & 0.0005115 & $0.2230 \%$ \\
\hline
\end{tabular}
values of these coefficients, and their percentage ratio for both pseudoscalar $\left(\eta_{c}, \eta_{b}\right)$, and vector $(J / \psi$ and $\Upsilon)$ mesons are given in Table 2 below, which justifies these terms being dropped.

Table 2: Numerical values of coefficients, $\Omega_{P}=m \Theta_{P} \omega_{q \bar{q}}^{2}$, and $\Omega_{P}^{\prime}=\frac{\Theta_{P}^{2}}{4} \omega_{q \bar{q}}^{4}$ associated with the terms involving $\bar{V}$ and $\bar{V}^{2}$ respectively, for pseudoscalar mesons $\eta_{c}$ and $\eta_{b}$ in RHS of Eqs.(22), and their percentage ratio, along with the corresponding values, and percentage ratio of $\Omega_{V}=m \Theta_{V} \omega_{q \bar{q}}^{2}$, and $\Omega_{V}^{\prime}=\frac{\Theta_{V}^{2}}{4} \omega_{q \bar{q}}^{4}$ for vector mesons, $J / \psi$ and $\Upsilon$ on RHS of Eqs.(29). The input parameters of our model are: $C_{0}=0.21$, $\omega_{0}=.15 \mathrm{GeV}$. QCD length scale $\Lambda=0.200 \mathrm{GeV} ., A_{0}=0.01$, and the input quark masses, $m_{c}=1.49 \mathrm{GeV}$. and $m_{b}=5.070 \mathrm{GeV}$.

To derive the mass spectrum, we put the spatial part, $\bar{V}$ in Eq.(34) into the equations Eq.(22), and Eq.(29) for pseudoscalar and vector mesons respectively. But the form of $V$ in Eq.(34) suggests that these equations have to be solved numerically. However, to solve these equations with $A_{0} \neq 0$, we follow an analytical procedure on lines of Ref.[36, 47], where we treat $\kappa$ as a "correction" factor due to small value of parameter, $A_{0}\left(A_{0}<<1\right)$, while we work in an approximate harmonic oscillator basis, due to its transparency in bringing out the dependence of mass spectral equations on the total quantum number 
$N$. (In this connection, we wish to mention that recently, harmonic oscillator basis has also been widely employed to study heavy quarkonia using a Light-front quark model [48].) The latter is achieved through the effective replacement, $\kappa=\left(1-A_{0} M^{2} \vec{\nabla}_{\hat{q}}^{2}\right)^{-\frac{1}{2}} \Rightarrow\left(1+2 A_{0}\left(N+\frac{3}{2}\right)\right)^{-\frac{1}{2}}$, (which is quite valid for heavy $c \bar{c}$ and $b \bar{b}$ systems), in Eq.(34) on lines of [36, 47]. With this, we can reduce Eq.(22) and Eq.(29) to equations of a simple quantum mechanical 3D- harmonic oscillator with coefficients depending on the hadron mass $M$ and total quantum number $N$. The wave function satisfies the 3D BSE for equal mass heavy pseudoscalar and vector mesons respectively as given below:

$$
\left(\frac{M^{2}}{4}-m^{2}-\widehat{q}^{2}\right) \phi_{P}(\hat{q})=\Theta_{P} m \omega_{q \bar{q}}^{2}\left[\frac{\vec{\nabla}_{\hat{q}}^{2}}{\sqrt{1+2 A_{0}\left(N+\frac{3}{2}\right)}}+\frac{C_{0}}{\omega_{0}^{2}}\right] \phi_{P}(\hat{q})
$$

and

$$
\left(\frac{M^{2}}{4}-m^{2}-\widehat{q}^{2}\right) \phi_{V}(\hat{q})=\Theta_{V} m \omega_{q \bar{q}}^{2}\left[\frac{\vec{\nabla}_{\hat{q}}^{2}}{\sqrt{1+2 A_{0}\left(N+\frac{3}{2}\right)}}+\frac{C_{0}}{\omega_{0}^{2}}\right] \phi_{V}(\hat{q})
$$

where, with use of dominant Dirac structures [39, 40, we can to a good approximation express, $\Theta_{P}=$ -4 for pseudoscalar states, and $\Theta_{V}=-2$ for vector states, and write Eqs.(35) and (36) in the same expression as:

$$
E_{P, V} \phi_{P, V}(\hat{q})=\left(-\beta_{P, V}^{4} \vec{\nabla}_{\hat{q}}^{2}+\hat{q}^{2}\right) \phi_{P, V}(\hat{q})
$$

where, $\vec{\nabla}_{\widehat{q}}^{2}=\frac{\partial^{2}}{\partial \widehat{q}^{2}}+\frac{2}{\hat{q}} \frac{\partial}{\partial \widehat{q}}-\frac{l(l+1)}{\widehat{q}^{2}}$, and $l=0,1,2, \ldots$ correspond to $S, P, D, \ldots$ states respectively. $E_{P, V}=$ $\frac{M^{2}}{4}-m^{2}+\frac{\beta_{P, V}^{4} C_{0}}{\omega_{0}^{2}} \sqrt{1+2 A_{0}\left(N+\frac{3}{2}\right)}$, and $\phi_{P, V}(\hat{q})$ are the eigen functions of Eq.(37) for equal mass heavy pseudoscalar, and vector quarkonia. The inverse range parameters for pseudoscalar and vector meson respectively are, $\beta_{P}=\left(4 \frac{m \omega_{q \bar{q}}^{2}}{\sqrt{1+2 A_{0}\left(N+\frac{3}{2}\right)}}\right)^{\frac{1}{4}}$, and $\beta_{V}=\left(2 \frac{m \omega_{q \bar{q}}^{2}}{\sqrt{1+2 A_{0}\left(N+\frac{3}{2}\right)}}\right)^{\frac{1}{4}}$ and are dependent on the input kernel and contains the dynamical information, and which only differ from each other due to spin-spin interactions. We can express Eq.(37) for the $l=0,2, \ldots$, states studied here as:

$$
\phi_{P, V}^{\prime \prime}(\widehat{q})+\frac{2}{\hat{q}} \phi_{P, V}^{\prime}(\widehat{q})+\frac{1}{\beta_{P, V}^{4}}\left[E_{P, V}-\frac{l(l+1) \beta_{P, V}^{4}}{\widehat{q}^{2}}-\widehat{q}^{2}\right] \phi_{P, V}(\widehat{q})=0 .
$$

Assuming the form of the solutions of the above equation as $\phi_{P, V}(\widehat{q})=h(\widehat{q}) \widehat{q}^{l} e^{-\frac{\hat{q}^{2}}{2 \beta^{2}}}$, where $\beta^{2}=\beta_{P, V}^{2}$, we obtain the equation,

$$
h^{\prime \prime}(\widehat{q})+\left(\frac{2 l}{\widehat{q}}+\frac{2}{\widehat{q}}-\frac{2 \widehat{q}}{\beta^{2}}\right) h^{\prime}(\widehat{q})+\left[\frac{E}{\beta^{4}}-\frac{2 l}{\beta^{2}}-\frac{3}{\beta^{2}}\right] h(\widehat{q})=0 .
$$

Making use of the power series method to solve this equation, we obtain the energy eigenvalues as: 


$$
\left(E_{P, V}\right)_{N}=2 \beta^{2}\left(N+\frac{3}{2}\right) ; N=2 n+l ; n=0,1,2, \ldots
$$

with the normalized forms of energy eigen functions for $l=0(S)$, and for $l=2(D)$ states derived as:

$$
\begin{aligned}
& \phi_{P(V)}(1 S, \hat{q})=\frac{1}{\pi^{3 / 4} \beta^{3 / 2}} e^{-\frac{\hat{q}^{2}}{2 \beta^{2}}}, \\
& \phi_{P(V)}(2 S, \hat{q})=\left(\frac{3}{2}\right)^{1 / 2} \frac{1}{\pi^{3 / 4} \beta^{3 / 2}}\left(1-\frac{2 \hat{q}^{2}}{3 \beta^{2}}\right) e^{-\frac{\hat{q}^{2}}{2 \beta^{2}}} \\
& \phi_{V}(1 D, \hat{q})=\left(\frac{4}{15}\right)^{1 / 2} \frac{1}{\pi^{3 / 4} \beta^{7 / 2}} \widehat{q}^{2} e^{-\frac{\hat{q}^{2}}{2 \beta^{2}}} \\
& \phi_{P(V)}(3 S, \hat{q})=\left(\frac{15}{8}\right)^{1 / 2} \frac{1}{\pi^{3 / 4} \beta^{3 / 2}}\left(1-\frac{20 \hat{q}^{2}}{15 \beta^{2}}+\frac{4 \hat{q}^{4}}{15 \beta^{4}}\right) e^{-\frac{\hat{q}^{2}}{2 \beta^{2}}}, \\
& \phi_{V}(2 D, \hat{q})=\left(\frac{14}{15}\right)^{1 / 2} \frac{1}{\pi^{3 / 4} \beta^{7 / 2}}\left(1-\frac{2 \widehat{q}^{2}}{7 \beta^{2}}\right) \widehat{q}^{2} e^{-\frac{\hat{q}^{2}}{2 \beta^{2}}}, \\
& \phi_{P(V)}(4 S, \hat{q})=\left(\frac{35}{16}\right)^{1 / 2} \frac{1}{\pi^{3 / 4} \beta^{3 / 2}}\left(1-\frac{210 \hat{q}^{2}}{105 \beta^{2}}+\frac{84 \hat{q}^{4}}{105 \beta^{4}}-\frac{8 \widehat{q}^{6}}{105 \beta^{6}}\right) e^{-\frac{\hat{q}^{2}}{2 \beta^{2}}} \\
& \phi_{V}(3 D, \hat{q})=\left(\frac{21}{10}\right)^{1 / 2} \frac{1}{\pi^{3 / 4} \beta^{7 / 2}}\left(1-\frac{36 \hat{q}^{2}}{63 \beta^{2}}+\frac{4 \hat{q}^{4}}{63 \beta^{4}}\right) \hat{q}^{2} e^{-\frac{\hat{q}^{2}}{2 \beta^{2}}} .
\end{aligned}
$$

We will use them for a description of the equal mass heavy pseudoscalar and vector mesons. In Fig.1, and Fig.2, we now give the plots of these normalized wave functions Vs. $\hat{q}$ (in Gev.) for different states of pseudoscalar $c \bar{c}$, and $b \bar{b}$ quarkonia. And in Fig.3, and Fig.4, we give the plots of these wave functions Vs. $\hat{q}$ (in Gev.) for different states of vector $c \bar{c}$, and $b \bar{b}$ quarkonia. It can be seen from these plots that the wave functions corresponding to $n S$, and $n D$ states have $n-1$ nodes.
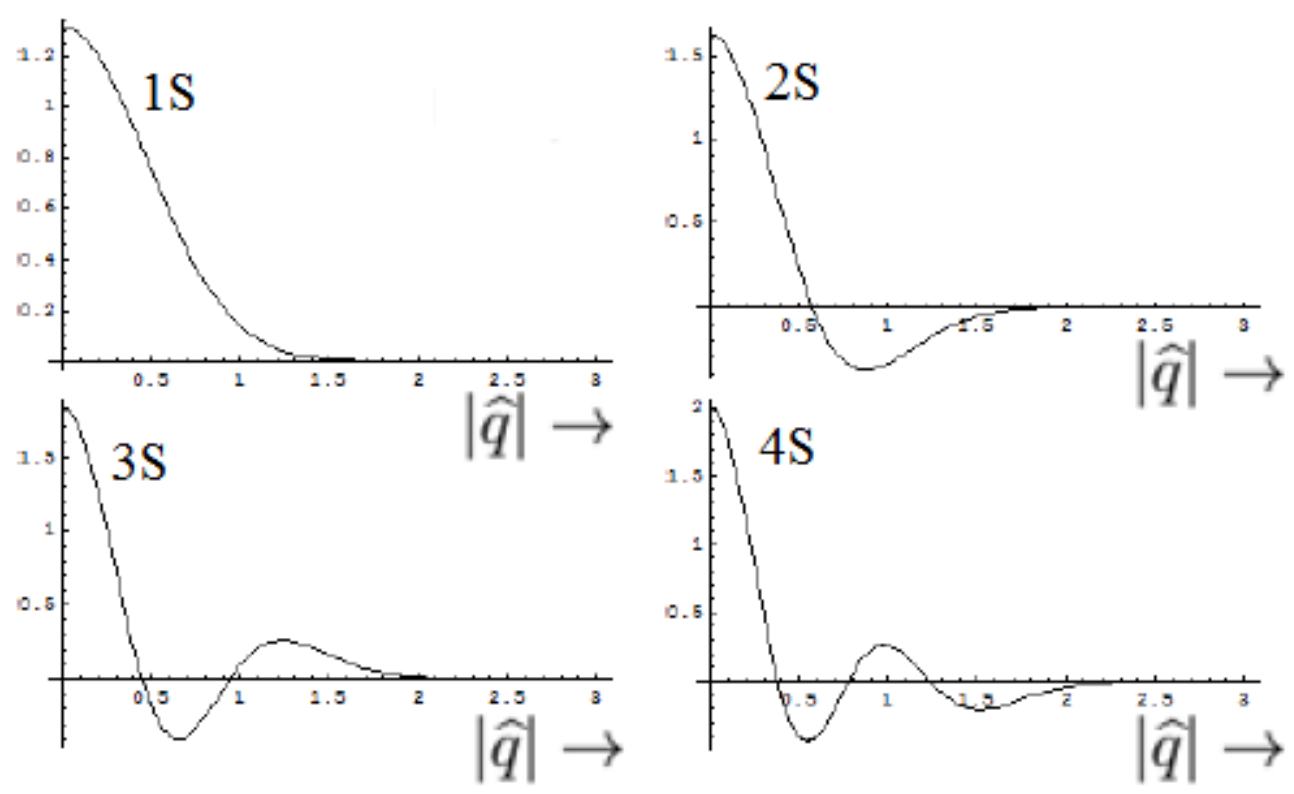

Figure 1: Plots of wave functions for states $(1 S, \ldots, 4 S) \mathrm{Vs} \widehat{q}$ (in Gev.) for pseudoscalar $c \bar{c}$ states. 

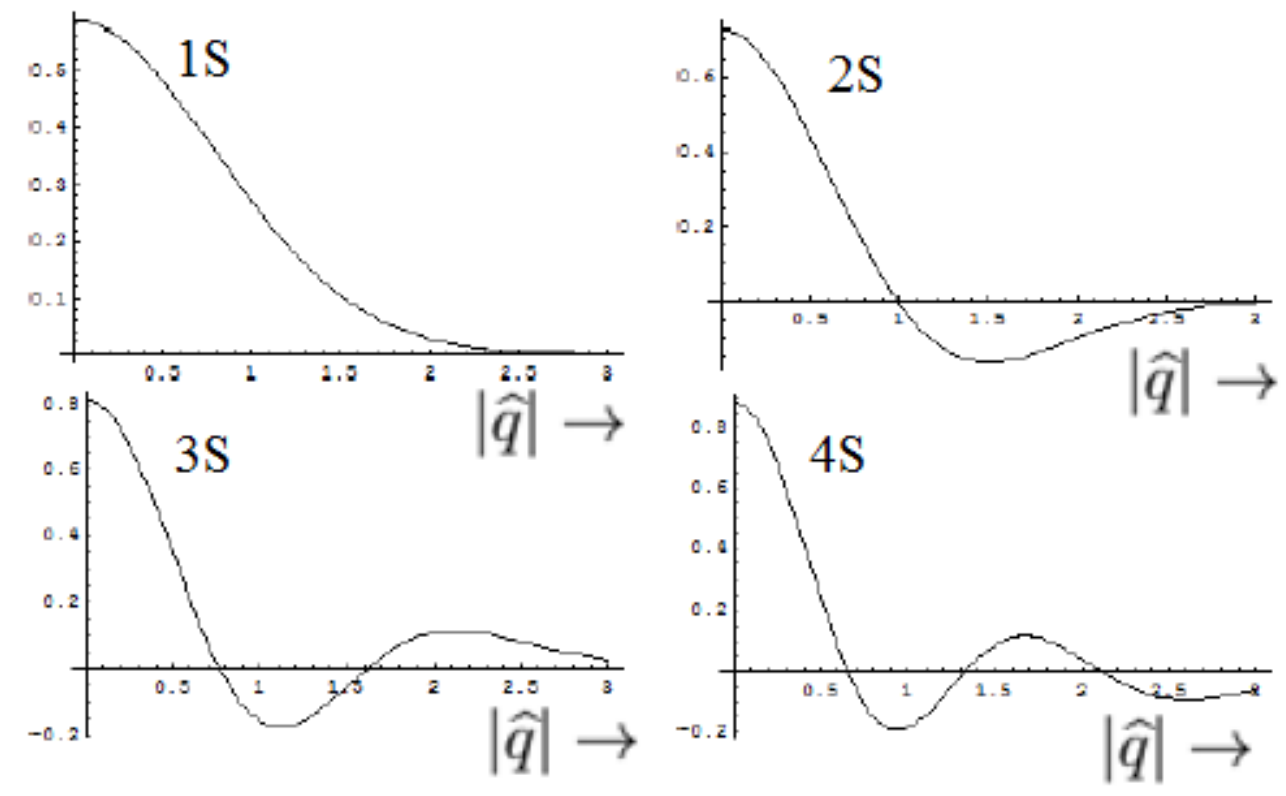

Figure 2: Plots of wave functions for states $(1 S, \ldots, 4 S)$ Vs $\widehat{q}$ (in Gev.) for pseudoscalar $b \bar{b}$ states.

The mass spectrum of ground $(1 \mathrm{~S})$ and excited states for equal mass heavy pseudoscalar $\left(0^{-+}\right)$and vector $\left(1^{--}\right)$mesons respectively is written as:

$$
\frac{1}{2 \beta_{P}^{2}}\left(\frac{M^{2}}{4}-m^{2}+\frac{C_{0} \beta_{P}^{4}}{\omega_{0}^{2}} \sqrt{1+2 A_{0}\left(N+\frac{3}{2}\right)}\right)=N+\frac{3}{2} ; N=2 n+l ; n=0,1,2, \ldots
$$

and

$$
\frac{1}{2 \beta_{V}^{2}}\left(\frac{M^{2}}{4}-m^{2}+\frac{C_{0} \beta_{V}^{4}}{\omega_{0}^{2}} \sqrt{1+2 A_{0}\left(N+\frac{3}{2}\right)}\right)=N+\frac{3}{2} ; N=2 n+l ; n=0,1,2 \ldots
$$

\begin{tabular}{lllllll}
\hline & BSE - CIA & Expt.[35] & Pot. Model[21] & QCD sum rule[12] & Lattice QCD [8] & [49] \\
\hline$M_{\eta_{c}(1 S)}$ & 2.9509 & $2.983 \pm 0.0007$ & 2.980 & $3.11 \pm 0.52$ & 3.292 & 2.981 \\
$M_{\eta_{c}(2 S)}$ & 3.7352 & $3.639 \pm 0.0013$ & 3.600 & & 4.240 & 3.635 \\
$M_{\eta_{c}(3 S)}$ & 4.4486 & & 4.060 & & 3.989 \\
$M_{\eta_{c}(4 S)}$ & 5.1048 & & 4.4554 & & & 4.401 \\
$M_{\eta_{b}(1 S)}$ & 9.0005 & $9.398 \pm 0.0032$ & 9.390 & $9.66 \pm 1.65$ & 7.377 & 9.398 \\
$M_{\eta_{b}(2 S)}$ & 9.7215 & $9.999 \pm 0.0028$ & 9.947 & & 8.202 & 9.990 \\
$M_{\eta_{b}(3 S)}$ & 10.4201 & & 10.291 & & & 10.329 \\
$M_{\eta_{b}(4 S)}$ & 11.0968 & & & & & 10.573 \\
\hline \hline
\end{tabular}

Table 3: Masses of ground and radially excited states of $\eta_{c}$ and $\eta_{b}$ (in GeV.) in present calculation (BSECIA) along with experimental data, and their masses in other models. 


\begin{tabular}{|c|c|c|c|c|c|c|}
\hline & BSE - CIA & Expt. 35] & Rel. Pot. Model[49] & Pot. Model[21] & BSE[52] & Lattice QCD [51] \\
\hline$M_{J / \psi(1 S)}$ & 3.0974 & $3.0969 \pm 0.000011$ & 3.096 & 3.0969 & 3.0969 & 3.099 \\
\hline$M_{\psi(2 S)}$ & 3.6676 & $3.6861 \pm 0.00034$ & 3.685 & 3.6890 & 3.686 & 3.653 \\
\hline$M_{\psi(1 D)}$ & 3.6676 & $3.773 \pm 0.00033$ & 3.783 & & 3.759 & \\
\hline$M_{\psi(3 S)}$ & 4.1945 & $4.03 \pm 0.001$ & 4.039 & 4.1407 & 4.065 & 4.099 \\
\hline$M_{\psi(2 D)}$ & 4.1945 & $4.191 \pm 0.005$ & 4.150 & & 4.108 & \\
\hline$M_{\psi(4 S)}$ & 4.6856 & $4.421 \pm 0.004$ & 4.427 & 4.5320 & 4.344 & \\
\hline$M_{\psi(3 D)}$ & 4.6856 & & 4.507 & & 4.371 & \\
\hline$M_{\psi(5 S)}$ & 5.1463 & & 4.837 & 4.8841 & 4.567 & \\
\hline$M_{\psi(4 D)}$ & 5.1463 & & 4.857 & & & \\
\hline$M_{\Upsilon(1 S)}$ & 9.6719 & $9.4603 \pm 0.00026$ & 9.460 & 9.4603 & 9.460 & \\
\hline$M_{\Upsilon(2 S)}$ & 10.1926 & $10.0233 \pm 0.00031$ & 10.023 & 9.9814 & 10.029 & \\
\hline$M_{\Upsilon(1 D)}$ & 10.1926 & & 10.154 & & 10.139 & \\
\hline$M_{\Upsilon(3 S)}$ & 10.6979 & $10.3552 \pm 0.00005$ & 10.355 & 10.3195 & 10.379 & \\
\hline$M_{\Upsilon(2 D)}$ & 10.6979 & & 10.435 & & 10.438 & \\
\hline$M_{\Upsilon(4 S)}$ & 11.1887 & $10.5794 \pm 0.0012$ & 10.586 & 10.5995 & 10.648 & \\
\hline$M_{\Upsilon(3 D)}$ & 11.1887 & & 10.704 & & 10.690 & \\
\hline$M_{\Upsilon(5 S)}$ & 11.6657 & $10.865 \pm 0.008$ & 10.869 & 10.8465 & 10.868 & \\
\hline$M_{\Upsilon(4 D)}$ & 11.6657 & & 10.949 & & & \\
\hline$M_{\Upsilon(6 S)}$ & 12.1296 & $11.019 \pm 0.008$ & 11.088 & 11.0713 & & \\
\hline$M_{\Upsilon(5 D)}$ & 12.1296 & & & & & \\
\hline
\end{tabular}

Table 4: Masses of ground, radially and orbitally excited states of heavy vector quarkonium, $J / \psi$ and $\Upsilon$ in BSE-CIA along with their masses in other models and experimental data (all units are in GeV). 

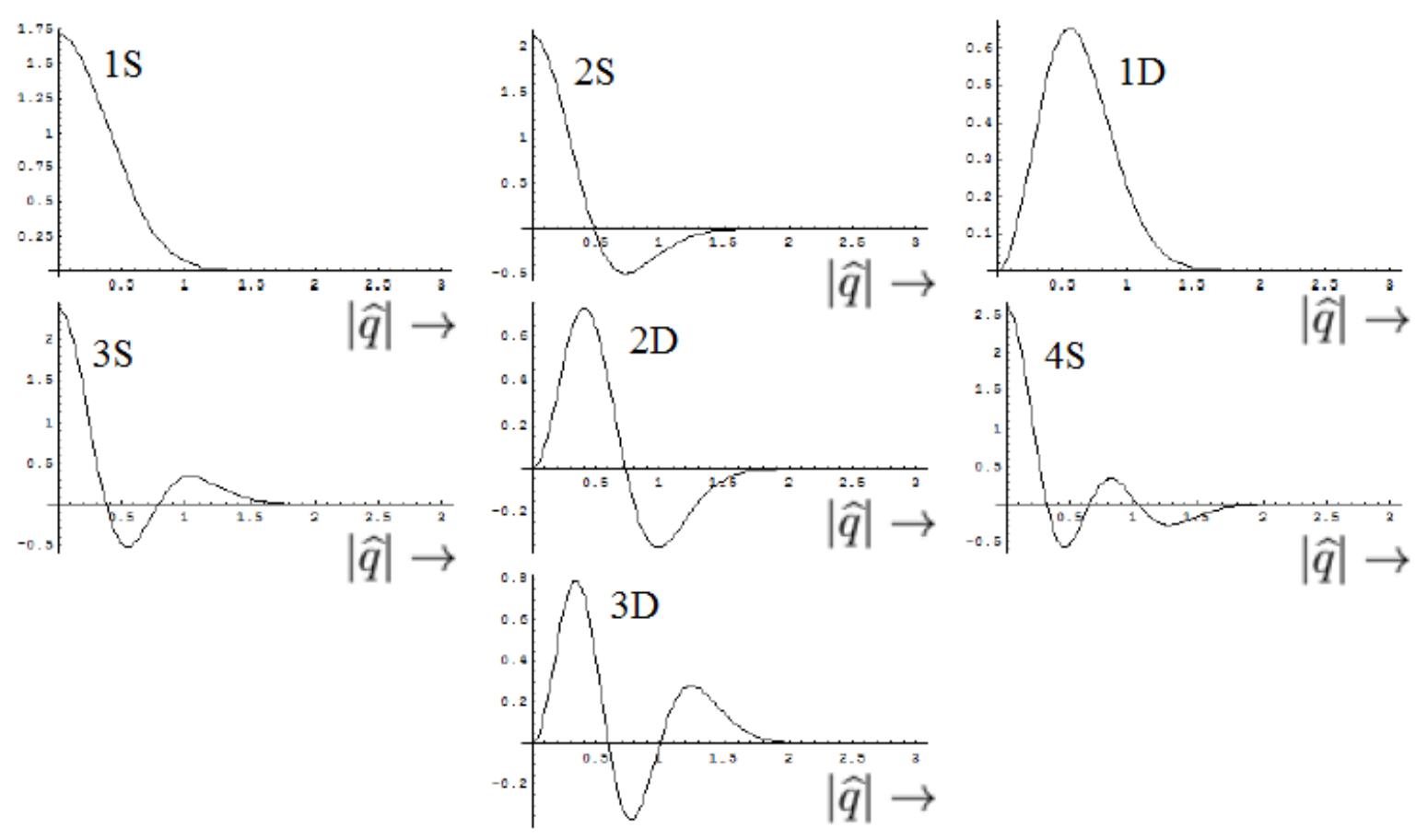

Figure 3: Plots of wave functions for states $(1 S, \ldots, 3 D)$ Vs $\widehat{q}$ (in Gev.) for vector $c \bar{c}$ states.

The input parameters of our model are: $C_{0}=0.21, \omega_{0}=.15 G e V$. QCD length scale $\Lambda=0.200 G e V$, $A_{0}=0.01$, and the input quark masses, $m_{c}=1.49 \mathrm{GeV}$. , and $m_{b}=5.070 \mathrm{GeV}$. The results of mass spectral predictions of heavy equal mass pseudoscalar and vector mesons for both ground and excited states with the above set of parameters is given in table 3 and 4 . We now present the calculation of the leptonic decay constants $f_{P}$ and $f_{V}$ for these equal mass heavy pseudoscalar and vector mesons, $\eta_{c}, \eta_{b}, J / \psi$ and $\Upsilon$.

\section{Leptonic Decays of equal mass Heavy pseudoscalar and vec- tor quarkonia}

We now do the calculation of decay constants of equal mass pseudoscalar and vector mesons such as, $\eta_{c}$, $\eta_{b}, J / \psi$ and $\Upsilon$, which are defined as [43, 50],

$$
\begin{aligned}
& i f_{P} P_{\mu} \equiv<0\left|\bar{Q} i \gamma_{\mu} \gamma_{5} Q\right| P> \\
& \quad f_{V} M \epsilon_{\mu}(P) \equiv<0\left|\bar{Q} \gamma_{\mu} Q\right| V(P)>
\end{aligned}
$$

The decay constants $f_{P}$ and $f_{V}$ thus can be evaluated through the loop diagram which gives the coupling of two-quark loop to the axial vector current and vector current respectively, and can be expressed as a quark-loop integral (for some of our recent works on leptonic decays in the framework of BSE, see, 

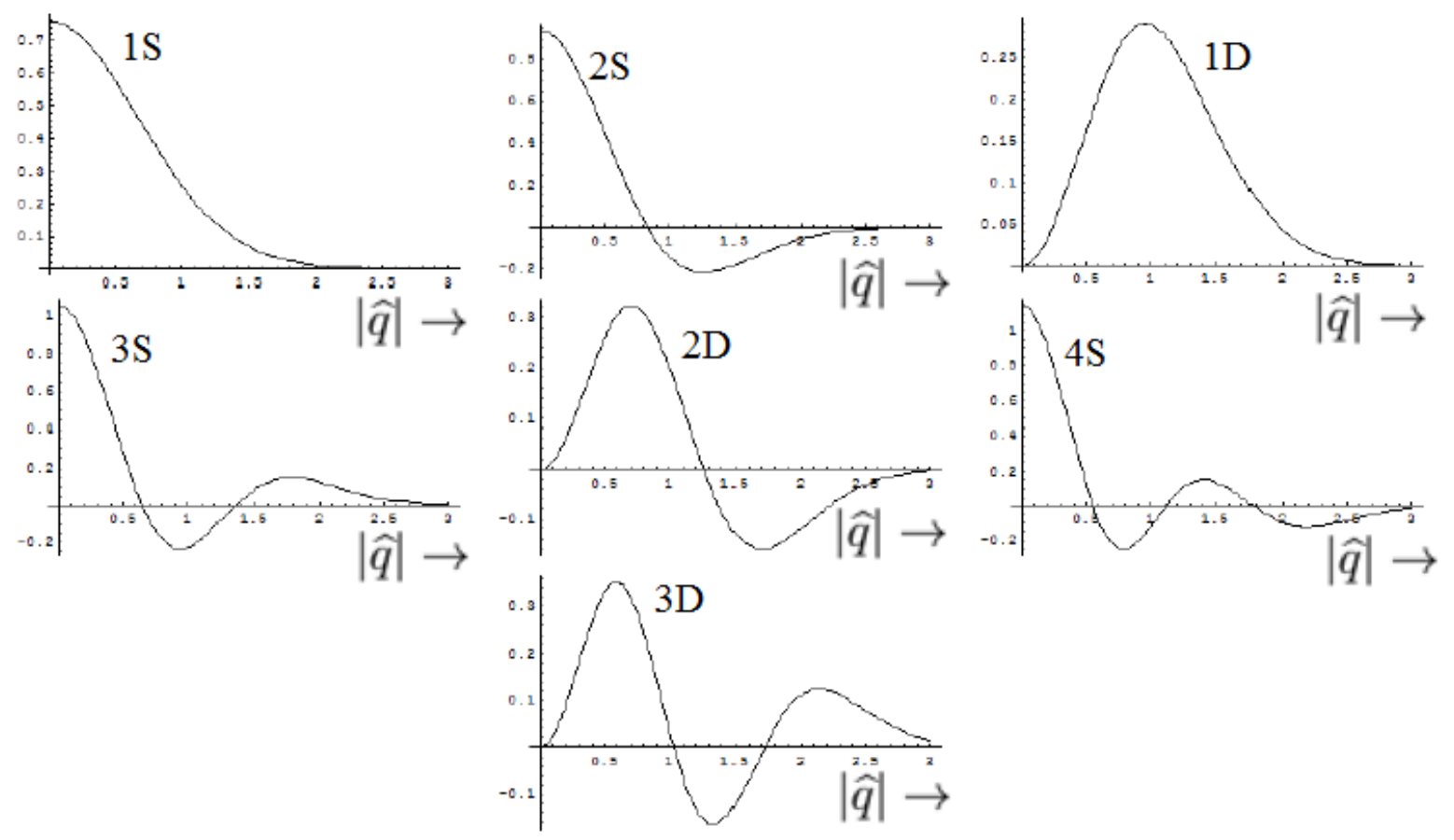

Figure 4: Plots of wave functions for states $(1 S, \ldots, 3 D)$ Vs $\widehat{q}$ (in Gev.) for vector $b \bar{b}$ states.

[38, 39, 40, 43]):

$$
\begin{aligned}
& f_{P} P_{\mu}=\sqrt{3} \int \frac{d^{4} q}{(2 \pi)^{4}} \operatorname{Tr}\left[\Psi^{P}(P, q) i \gamma_{\mu} \gamma_{5}\right] \\
& f_{V} M \epsilon_{\mu}=\sqrt{3} \int \frac{d^{4} q}{(2 \pi)^{4}} \operatorname{Tr}\left[\Psi^{V}(P, q) i \gamma_{\mu}\right]
\end{aligned}
$$

where $\epsilon_{\mu}$ is polarization vector of vector meson satisfying $\epsilon . P=0$. These equations can be reduced to 3D forms by defining the 3D wave function, $\psi(\hat{q})=-\int \frac{M d \sigma}{2 \pi i} \Psi(P, q)$. Thus we can write Eq.(45) as:

$$
\begin{gathered}
f_{P} P_{\mu}=\sqrt{3} \int \frac{d^{3} \hat{q}}{(2 \pi)^{3}} \operatorname{Tr}\left[\psi^{P}(\hat{q}) \gamma_{\mu} \gamma_{5}\right] \\
f_{V} M \epsilon_{\mu}=\sqrt{3} \int \frac{d^{3} \hat{q}}{(2 \pi)^{3}} \operatorname{Tr}\left[\psi^{V}(\hat{q}) \gamma_{\mu}\right]
\end{gathered}
$$

where (following Eq.(23) and Eq.(30)), the complete 3D Bethe-Salpeter wave function of state $\left(0^{-+}\right)$and $\left(1^{--}\right)$is rewritten as:

$$
\begin{aligned}
& \psi^{P}(\hat{q})=N_{P} \phi_{P}(\hat{q})\left[M+\not P+\frac{\not \underline{P}}{m}\right] \gamma_{5}, \\
& \psi^{V}(\hat{q})=N_{V} \phi_{V}(\hat{q})\left[M \notin+\hat{q} \cdot \epsilon \frac{M}{m}+\notin \not P+\frac{\not P \hat{q} . \epsilon}{m}-\frac{\not P \notin \not}{m}\right],
\end{aligned}
$$

where $N_{P}$ and $N_{V}$ are the standard BS normalizers which enter into the BS wave function, and $m$ and $M$ are the masses of quarks and the corresponding meson respectively.

The 4D BS normalizer $N_{P}$ and $N_{V}$ are evaluated from the current conservation condition:

$$
2 i P_{\mu}=\int \frac{d^{4} q}{(2 \pi)^{4}} \operatorname{Tr}\left\{\bar{\Psi}(P, q)\left[\frac{\partial}{\partial P_{\mu}} S_{F}^{-1}\left(p_{1}\right)\right] \Psi(P, q) S_{F}^{-1}\left(-p_{2}\right)\right\}+(1 \rightleftharpoons 2) .
$$


Carrying out derivatives of inverse quark propagators of constituent quarks with respect to total hadron momentum $P_{\mu}$, evaluating trace over products of gamma matrices, following usual steps, we then express the above equation in terms of the integration variables $\hat{q}$ and $\sigma$. Noting that the $4 \mathrm{D}$ volume element $d^{4} q=d^{3} \widehat{q} M d \sigma$, we then perform the contour integration in the complex $\sigma$-plane by making use of the corresponding pole positions. Then integration over the variable $\hat{q}$ is finally performed to extract out the numerical results for $N_{P}$ and $N_{V}$ for different equal mass pseudoscalar and vector mesons. The above equation due to the orthogonality condition, $P . \widehat{q}=0$ and $P . \epsilon=0$ reduces to a simple forms given below for equal mass pseudoscalar and vector mesons:

$$
N_{P}^{-2}=\frac{16 M}{m} \int \frac{d^{3} \hat{q}}{(2 \pi)^{3}} \frac{\hat{q}^{2}}{\omega} \phi_{P}^{2}(\hat{q})
$$

and

$$
N_{V}^{-2}=16 M m \int \frac{d^{3} \hat{q}}{(2 \pi)^{3}} \frac{\hat{q}^{2}}{\omega^{3}} \phi_{V}^{2}(\hat{q}) .
$$

Here, in the above equations for decay constants and the BS normalizers, the wave functions, $\phi_{P}$ and $\phi_{V}$ represent the eigenfunctions of the pseudoscalar and vector mesons obtained by solving the full Salpeter equation. Their algebraic expressions are taken from Eq. (41), and are then employed to calculate $f_{P}$ and $f_{V}$ as well as the BS normalizers $N_{P}$, and $N_{V}$ for ground as well as the excited states of $\eta_{c}, \eta_{b}, J / \psi$, and $\Upsilon$. The numerical values of BS normalizers of all these states are given in Table 5 below.

\begin{tabular}{lllll}
\hline & $N_{\eta_{c}}$ & $N_{\eta_{b}}$ & $N_{J / \psi}$ & $N_{\Upsilon}$ \\
\hline $1 S$ & 5.2239 & 5.7441 & 5.9102 & 6.4666 \\
$2 S$ & 3.1632 & 3.6679 & 3.6788 & 4.1743 \\
$1 D$ & & & 3.6233 & 4.1534 \\
$3 S$ & 2.3845 & 2.8657 & 2.8317 & 3.2904 \\
$2 D$ & & & 2.8079 & 3.2805 \\
$4 S$ & 2.1531 & 2.4103 & 2.3591 & 2.7881 \\
$3 D$ & & & 2.3462 & 2.7058 \\
\hline
\end{tabular}

Table 5: Numerical values of BS normalizers for ground state and excited states of $\eta_{c}, \eta_{b}, J / \psi$ and $\Upsilon$ (in $\mathrm{GeV}$ units) in present calculation.

The decay constant from Eq.(46) becomes:

$$
\begin{aligned}
& f_{P} P_{\mu}=\sqrt{3} N_{P} \int \frac{d^{3} \hat{q}}{(2 \pi)^{3}} \phi_{P}(\hat{q}) \operatorname{Tr}\left[\left(M+\not P+\frac{\not \not P}{m}\right) \gamma_{\mu}\right] \\
& f_{V} M \epsilon_{\mu}=\sqrt{3} N_{V} \int \frac{d^{3} \hat{q}}{(2 \pi)^{3}} \phi_{V}(\hat{q}) \operatorname{Tr}\left[\left(M \notin+\hat{q} \cdot \epsilon \frac{M}{m}+\notin \not P+\frac{\not P \hat{q} . \epsilon}{m}-\frac{\not P \notin \not \mid}{m}\right) \gamma_{\mu}\right]
\end{aligned}
$$


Evaluating trace over $\gamma$-matrices, and carrying out integration over $d^{3} \hat{q}$, we obtain:

$$
\begin{aligned}
& f_{P}=4 \sqrt{3} N_{P} \int \frac{d^{3} \hat{q}}{(2 \pi)^{3}} \phi_{P}(\hat{q}) \\
& f_{V}=4 \sqrt{3} N_{V} \int \frac{d^{3} \hat{q}}{(2 \pi)^{3}} \phi_{V}(\hat{q}) .
\end{aligned}
$$

\begin{tabular}{llllll}
\hline & BSE - CIA & Expt.[34] & Lattice QCD [6] & Pot. Model[21] & QCD sum rule[12] \\
\hline$f_{\eta_{c}(1 S)}$ & 0.4044 & $0.335 \pm 0.075$ & 0.3928 & 0.471 & $0.260 \pm 0.075$ \\
$f_{\eta_{c}(2 S)}$ & 0.3308 & & & 0.374 & \\
$f_{\eta_{c}(3 S)}$ & 0.2908 & & & 0.332 & \\
$f_{\eta_{b}(1 S)}$ & 1.0168 & 0.667 & 0.834 & $0.251 \pm 0.072$ \\
$f_{\eta_{b}(2 S)}$ & 0.8066 & & 0.567 & \\
$f_{\eta_{b}(3 S)}$ & 0.7134 & & 0.508 & \\
\hline \hline
\end{tabular}

Table 6: Leptonic decay constants, $f_{P}$ of ground state $(1 \mathrm{~S})$ and excited state $(2 \mathrm{~S})$ and $(3 \mathrm{~S})$ of $\eta_{c}$ and $\eta_{b}$ (in $\mathrm{GeV}$.) in present calculation (BSE-CIA) along with experimental data, and their masses in other models.

The calculated values of decay constants are given in Tables 6 and 7 . We next calculate the decay widths for two photon and two gluon decays of pseudoscalar quarkonia $\eta_{c}$ and $\eta_{b}$ and their radially excited states in the next section.

\section{Two photon and two gluon decays of pseudoscalar quarko- nium}
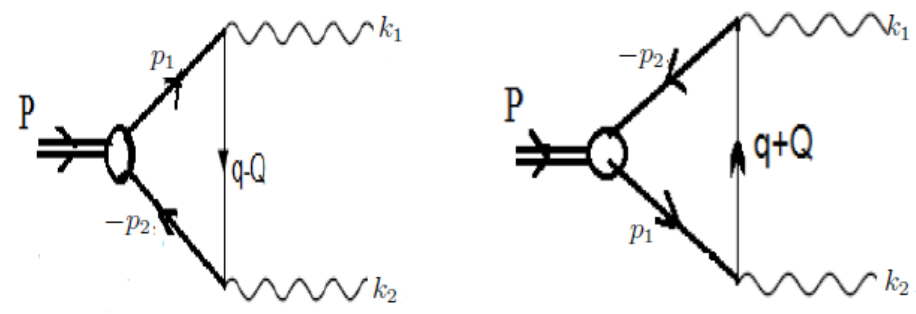

Figure 5: Diagrams contributing to process $P \rightarrow \gamma \gamma$. The second diagram is obtained from the first diagram by reversing the direction of internal fermion lines.

The two-photon decays of these states have been the subject of numerous studies aimed at further understanding the accuracy of theoretical models of the charmonium and bottomonium systems based on 


\begin{tabular}{lllllll}
\hline & BSE - CIA & Expt.[35] & Pot. Model[21] & BSE [52] & LatticeQCD[50] & Light front model[48] \\
\hline$f_{J / \psi(1 S)}$ & 0.3745 & $0.411 \pm .007$ & 0.317 & $0.459 \pm .028$ & $0.399 \pm 0.004$ & \\
$f_{\psi(2 S)}$ & 0.2953 & $0.279 \pm .008$ & 0.253 & $0.364 \pm .024$ & $0.143 \pm 0.081$ & 0.2474 \\
$f_{\psi(1 D)}$ & 0.2897 & $0.210 \pm 0.00024$ & & $0.243 \pm .017$ & & \\
$f_{\psi(3 S)}$ & 0.2610 & $0.174 \pm .018$ & 0.226 & $0.319 \pm .022$ & & \\
$f_{\psi(2 D)}$ & 0.2348 & $0.1424 \pm 0.0033$ & & $0.157 \pm .011$ & & \\
$f_{\psi(4 S)}$ & 0.2399 & $0.1608 \pm 0.0016$ & & $0.288 \pm .018$ & \\
$f_{\psi(3 D)}$ & 0.2570 & $0.1424 \pm 0.0033$ & & $0.157 \pm .011$ & \\
$f_{\Upsilon(1 S)}$ & 0.9005 & $0.708 \pm .008$ & 0.645 & $0.498 \pm .020$ & \\
$f_{\Upsilon(2 S)}$ & 0.6072 & $0.482 \pm .010$ & 0.439 & $0.366 \pm .027$ & & \\
$f_{\Upsilon(1 D)}$ & 0.5972 & & & 0.1922 & \\
$f_{\Upsilon(3 S)}$ & 0.5446 & $0.346 \pm .050$ & 0.393 & $0.304 \pm .027$ & & \\
$f_{\Upsilon(2 D)}$ & 0.5755 & & & $0.155 \pm .011$ & & \\
$f_{\Upsilon(4 S)}$ & 0.5859 & $0.3406 \pm .00037$ & & $0.259 \pm .022$ & & \\
$f_{\Upsilon(3 D)}$ & 0.6274 & & & $0.155 \pm .011$ & & \\
\hline
\end{tabular}

Table 7: Leptonic decay constants, $f_{V}$ of ground state (1S) and excited state $(2 \mathrm{~S})$ and $(3 \mathrm{~S})$ heavy vector quarkonium, $J / \psi$ and $\Upsilon$ in BSE-CIA along with their masses in other models and experimental data (all units are in $\mathrm{GeV}$ ). 
the available data. The process, $P \rightarrow \gamma \gamma$ proceeds through the famous quark triangle diagrams shown in Fig.5. In this figure the second diagram is obtained from the first one by reversing the directions of the internal fermion lines in the quark loop. Let, $k_{1,2}$, and $\epsilon_{1,2}$ be the momenta and polarization vectors of the two outgoing photons. Let, $p_{1}$ and $p_{2}$ be the momenta of constituent quark and anti-quark constituting the hadron with the total momentum $P=p_{1}+p_{2}$ and relative momentum $q_{\mu} . \Psi(P, q)$ is the $4 \mathrm{D}$ hadron Bethe-Salpeter wave function. For sake of convenience, we introduce the relative momentum of the two outgoing photons: $2 Q=k_{1}-k_{2}$. In terms of $P$ and $Q$, we can express the momenta of the outgoing photons as: $k_{1,2}=\frac{1}{2} P \pm Q$. The momenta of the third quark in the two diagrams can be expressed as: $p=q-Q$ and $p=q+Q$ respectively.

The amplitude for the process in Fig.5 can be expressed as the sum of amplitudes for the two diagrams in this figure as:

$$
M_{f i}(P \rightarrow \gamma \gamma)=i \sqrt{3}\left(i e_{q}\right)^{2} \int \frac{d^{4} q}{(2 \pi)^{4}} \operatorname{Tr}\left\{\Psi^{P}(P, q)\left[\notin_{1} S_{F}(q-Q) \notin_{2}+\notin_{2} S_{F}(q+Q) \notin_{1}\right]\right\}
$$

$e_{q}=\frac{+2}{3} e$ for charm quark and $e_{q}=\frac{-1}{3} e$ for bottom quark, $S_{F}(q \mp Q)$ are the quark and anti-quark propagators which are given as:

$$
S_{F}(q \mp Q)=\frac{(\not q \mp \not Q) \pm m}{(q \mp Q)^{2} \mp m^{2}} .
$$

Now for heavy hadrons like $c \bar{c}$ and $b \bar{b}$, where the system is basically non-relativistic, it is convenient to take the internal momentum $q<<M$, and hence, $q^{2}<<Q^{2}$, where it can be easily seen that $Q^{2}=\frac{M^{2}}{4}$. We now make use of the fact that the $4 \mathrm{D}$ volume element, $d^{4} q=d^{3} \hat{q} M d \sigma$, and use the relationship between $3 \mathrm{D}$ and $4 \mathrm{D}$ BS wave functions. And since the rest of the integrand does not involve $q$, we can express the amplitude in the above Eq.(53) as:

$$
M_{f i}(P \rightarrow \gamma \gamma)=\frac{i \sqrt{3}\left(i e_{q}\right)^{2}}{m^{2}+M^{2} / 4} \int \frac{d^{3} \hat{q}}{(2 \pi)^{3}} \operatorname{Tr}\left\{\psi^{P}(\hat{q})\left[\notin_{1}(m+i \not) \notin_{2}+\notin_{2}(m-i \phi) \notin_{1}\right]\right\} .
$$

Now for a pseudoscalar meson with state $J^{P C}=0^{-+}$, the general relativistic Salpeter wave function $\psi(\hat{q})$ can be written as:

$$
\psi^{P}(\hat{q})=N_{P} \phi_{P}(\hat{q})\left[M+\not P+\frac{\not{ }_{1} P}{m}\right] \gamma_{5}
$$

where $N_{P}$ is the standard BS normalizer which enters into the BS wave function, and $m$ and $\mathrm{M}$ are the masses of quarks and the corresponding quarkonium respectively. The wave function $\phi_{P}(\hat{q})$, represents the eigenfunction of the Pseudoscalar meson obtained by solving the full instantaneous Bethe-Salpeter equation. For ground state $(1 S)$ mesons, $\phi_{P}(\widehat{q})=e^{-\frac{\hat{q}^{2}}{2 \beta^{2}}}$. The wave functions for excited states can similarly be expressed, while the BS normalizer $N_{P}$ is given as in Eq.(49). Putting the Salpeter wave function $\psi^{P}(\hat{q})$ into the amplitude in Eq.(55), and performing the trace over the gamma matrices, we 
obtain:

$$
M_{f i}(P \rightarrow \gamma \gamma)=\left[F_{P}\right] \varepsilon_{\mu \nu \alpha \sigma} P_{\mu} \epsilon_{1 \nu} Q_{\alpha} \epsilon_{2 \sigma}
$$

where $F_{P}$ is the radiative decay constant for two-photon decays of pseudoscalar meson and is expressed as:

$$
F_{P}=\frac{8 \sqrt{3} e_{q}^{2}}{m^{2}+M^{2} / 4} N_{P} \int \frac{d^{3} \hat{q}}{(2 \pi)^{3}} \phi_{P}(\hat{q})
$$

The decay width for $P \rightarrow \gamma \gamma$ is related to the corresponding decay constant, $F_{P}$ by the expression:

$$
\Gamma_{P \rightarrow \gamma \gamma}=\frac{\left|F_{P}\right|^{2} M^{3}}{64 \pi}
$$

The numerical results of $\Gamma_{P \rightarrow \gamma \gamma}$ are given in the Table 8. We now work out the process of two-gluon decays of pseudoscalar quarkonia.

\begin{tabular}{llllllll}
\hline & BSE-CIA & Expt.[35] & BSE[29] & BSE[16] & RQM[26] & [30] & Pot.Model[21] \\
\hline$\Gamma_{\eta_{c}(1 S) \rightarrow \gamma \gamma}$ & 7.9178 & $7.2 \pm 1.2$ & 7.14 & 3.5 & 5.5 & $7.5-10$ & 11.17 \\
$\Gamma_{\eta_{c}(2 S) \rightarrow \gamma \gamma}$ & 5.7889 & & 4.44 & 1.38 & 1.8 & $3.5-4.5$ & 8.48 \\
$\Gamma_{\eta_{c}(3 S) \rightarrow \gamma \gamma}$ & 0.2995 & & & 0.94 & & & 7.57 \\
$\Gamma_{\eta_{b}(1 S) \rightarrow \gamma \gamma}$ & 0.7376 & & 0.384 & 0.22 & 0.35 & 0.560 & 0.58 \\
$\Gamma_{\eta_{b}(2 S) \rightarrow \gamma \gamma}$ & 0.5076 & & 0.191 & 0.11 & 0.15 & 0.269 & 0.29 \\
$\Gamma_{\eta_{b}(3 S) \rightarrow \gamma \gamma}$ & 0.4261 & & & 0.084 & 0.10 & 0.208 & 0.24 \\
\hline
\end{tabular}

Table 8: Two-photon decay widths of ground state (1S) and excited state (2S) and (3S) pseudoscalar mesons, $\eta_{c}$ and $\eta_{b}$ in present calculation (BSE-CIA) along with their masses in other models and experimental data (all values are in units of Kev).

The two-gluon decay width gives information on the total width of the corresponding quarkonium. The diagrams for two-gluon decays of quarkonium can be easily obtained from the diagrams for two photon decays of pseudoscalar quarkonia (in Fig.5), with a simple replacement of photons by gluons, and hence, the two quark-photon vertices with the corresponding quark-gluon vertices. This would lead to the replacement: $\alpha \rightarrow \frac{3}{2 \sqrt{2}} \alpha_{s}$ [41], in the expression for $F_{P}$ in Eq.(58), entering into the two-photon decay width formula, with $\alpha_{s}$ being the QCD coupling constant. Here we have taken $e_{q}=+\frac{2}{3} \sqrt{4 \pi \alpha}$ for c-quark, and $e_{q}=-\frac{1}{3} \sqrt{4 \pi \alpha}$ for b-quark. The results are shown in Table 9. 


\begin{tabular}{lllllll}
\hline & BSE-CIA & Expt. & BSE[29] & Pot.Model[21] & BSE[41] & Pot. Model[46] \\
\hline$\Gamma_{\eta_{c}(1 S) \rightarrow g g}$ & 13.0699 & $26.7 \pm 3.0$ & 19.6 & 32.44 & 10.57 & 9.010 \\
$\Gamma_{\eta_{c}(2 S) \rightarrow g g}$ & 9.5340 & $14.0 \pm 7.0$ & 12.1 & 24.64 & 5.94 & \\
$\Gamma_{\eta_{c}(3 S) \rightarrow g g}$ & 4.4123 & & & 21.99 & & \\
$\Gamma_{\eta_{b}(1 S) \rightarrow g g}$ & 10.8646 & & 6.98 & 13.72 & 12.39 & \\
$\Gamma_{\eta_{b}(2 S) \rightarrow g g}$ & 7.4766 & 3.47 & 6.73 & 5.61 & \\
$\Gamma_{\eta_{b}(3 S) \rightarrow g g}$ & 6.2763 & & & 5.58 & 4.11 & \\
\hline \hline
\end{tabular}

Table 9: Two-gluon decay widths of ground state (1S) and excited state (2S) and (3S) pseudoscalar mesons, $\eta_{c}$ and $\eta_{b}$ in present calculation (BSE-CIA) along with their masses in other models and experimental data (all values are in units of Mev).

\section{$6 \quad$ Numerical Results and Discussions}

We have employed a $3 \mathrm{D}$ reduction of $\mathrm{BSE}$ (with a $4 \times 4$ representation for two-body $(q \bar{q}) \mathrm{BS}$ amplitude) under Covariant Instantaneous Ansatz (CIA) for deriving the algebraic forms of the mass spectral equations whose analytic solutions (both eigen functions and eigen values), in Eq.(42-43), lead to mass spectra for ground and excited states of both pseudoscalar $\left(\eta_{c}\right.$, and $\left.\eta_{b}\right)$ and vector $(J / \Psi$, and $\Upsilon)$ quarkonia, in an approximate harmonic oscillator basis. The masses, and the eigen functions so obtained are used for calculating the leptonic decay constants, weak decay constants, two-photon decay widths, and two-gluon decay widths for ground and excited states of these pseudoscalar and vector quarkonia.

The mass spectrum calculated in this BSE framework for $(1 S, \ldots, 4 S)$ states of $\eta_{c}$ and $\eta_{b}$, while for $(1 S, 2 S, 1 D, 3 S, 2 D, \ldots, 5 D)$ states of $J / \psi$ and $\Upsilon$ are shown in Table 3 and 4 respectively. All numerical calculations have been done using Mathematica. We selected the best set of 6 input parameters, that gave good matching with data for masses of ground and excited states of $\eta_{c}, \eta_{b}, J / \Psi$ and $\Upsilon$ mesons. This input parameter set was found to be $C_{0}=0.21, \omega_{0}=0.15 \mathrm{GeV} ., \Lambda=0.200 \mathrm{GeV}$, and $A_{0}=0.01$, along with the input quark masses $m_{c}=1.490 \mathrm{GeV}$. and $m_{b}=5.070 \mathrm{GeV}$. The same set of parameters above was used to calculate the leptonic decay constants of $\eta_{c}, \eta_{b}, J / \psi$, and $\Upsilon$, as well as the two-photon and two-gluon decay widths of $\eta_{c}$, and $\eta_{b}$. However the experimental data on masses and decay constants/ decay widths of many of these states is not yet currently available. The results obtained for masses of ground and radially excited states of $\eta_{c}, \eta_{b}$, shown in Table 3 , are in reasonable agreement with experiment. However, a wide range of variation in masses of various states in different models such as Lattice QCD model [8], and QCD sum rule model [12] can be seen in Table 3.

As regards the mass spectral predictions for vector mesons is concerned, we have listed the values for 
$M_{J / \psi}$ for $1 S, 2 S, 1 D, \ldots 5 S$, and for $M_{\Upsilon}$ for $1 S, 2 S, 1 D, \ldots, 5 D$ in Table 4 . Many other states such as $3 D$, $4 D$, and $5 D$ for $J / \Psi$ are not yet experimentally available. The same holds true for all the $D$ states of $\Upsilon$. For vector $c \bar{c}$ quarkonia, the masses of ground and excited states are very close to central values of data. However the $1 S$, and $2 S$ states of $b \bar{b}$ are somewhat over estimated from central values of data. The disagreement with data increases as we go to the higher excited states of $b \bar{b}$. This is due to the fact that we did not incorporate the one-gluon-exchange (OGE) effects in the kernel, and used only the confining part of interaction as in our Eq.(31) (taking analogy from [19, 23, 43, 46] for heavy quarkonia). Our results reflect the fact that the OGE term becomes more and more important as we go to very heavy $b \bar{b}$ quarkonia, where the distance between the quark and the anti-quark will be tiny, and its contribution to their mass spectra will be substantial. Further, there is a degeneracy in the masses of $S$ and $D$ states with the same principal quantum number $N$ for $J / \Psi$ and $\Upsilon$. The inclusion of OGE terms in the potential will also lift up the degeneracy in these states and also bring masses of $b \bar{b}$ vector quarkonia closer to experiment.

However, we wish to mention that in this paper, our main emphasis was to show that this problem of $4 \times 4$ BSE under heavy quark approximation can indeed be handled analytically for both masses, as well as the wave functions in an approximate harmonic oscillator basis. The analytical forms of wave functions obtained as solutions of mass spectral equations (that are derived from 3D BSE), were then used to calculate the decay constants and decay widths for various processes involving these quarkonia. We next intend to incorporate the OGE effects perturbatively into our mass spectral equations, using our wave functions so obtained in Eqs.(41) as unperturbed wave functions in this study.

As regards our results for decay constants is concerned, it is seen that our calculated $f_{P}$ value of ground state is $f_{\eta_{c}(1 S)}=0.4044 \mathrm{GeV}$., and is within the error bars of its experimental value, $0.335 \pm 0.075 \mathrm{Gev}$ [34. However a wide range of predictions of decay constant values for all states can be seen in different models in Table 6. The experimental data for many of these states is not yet available. However, it is observed that the decay constants keep decreasing as one goes from $(1 S)$ to $(3 S)$ states for both $\eta_{c}$ and $\eta_{b}$ mesons. This also signifies that the instability of these states increases with increase in radial quantum number $N$. This trend is similar to the trend observed in a recent potential model calculation [21] of decay constants of $\eta_{c}$ and $\eta_{b}$.

Our results for leptonic decay constants of ground and excited states of $J / \psi$ and $\Upsilon$ are listed in Table 7. Our decay constants for $J / \psi$ and $\Upsilon$ for excited states are somewhat on the higher side in comparison to central values of data for these states. However, the decay constant $f_{V}$ values of various models again show a very wide range of variation as can be seen from Table 7.

Then, with the same set of parameters, we calculate the two-photon radiative decay widths of the ground and first two radially excited states of $\eta_{c}$ and $\eta_{b}$ mesons. The two photon decay widths of our model 
are listed in Table 8. Our result for two-photon decay width of ground state of $\eta_{c}$ is $\Gamma_{\eta_{c}}(1 S)=7.9178 \mathrm{KeV}$., and is within the error bars of data, $\Gamma_{\eta_{c}(1 S)}(\operatorname{Exp})=.7.2 \pm 1.2 \mathrm{KeV}$ [35]. We have compared our results with those of other models, though data on many of these is not yet available. The two photon decay widths of other models also show a wide range of variation.

We then calculate the two-gluon decay widths in our model for ground and radially excited states of $\eta_{c}$ and $\eta_{b}$, which are shown in Table 9. The two-gluon decay process accounts for a substantial portion of hadronic decay widths for states below $c \bar{c}$ or $b \bar{b}$ threshold. However, as pointed out in [41], due to significant contributions from radiative corrections as well as from three-gluon decays, the two gluon mode does not give the complete picture. Our results on two-gluon widths are thus smaller than the hadronic widths of $\eta_{c}$ and $\eta_{b}$ states. However as can be seen from Table 9, the results of two-gluon decay widths in various models again show a wide range of variations.

However, as mentioned earlier, our main emphasis in this paper was to show that this problem of $4 \times 4$ BSE under heavy quark approximation can indeed be handled analytically for both masses, as well as the wave functions for $l=0$, and $l=2$ states. The validity of heavy quark approximation for quarkonia is due to the fact that not only the relative momentum between heavy quarks in the bound states is considered small, but also these quarks are treated as almost on mass shell [53], which is justified for calculation of low energy properties like the mass spectrum, and the decays of quarkonia $(c \bar{c}$ and $b \bar{b}$ systems). With the above approximation, that is quite justifiable and well under control, in the context of heavy quark systems, we have been able to give analytical solutions of mass spectral equations Eqs.(35-36) of both pseudoscalar and vector quarkonia, giving us a much deeper insight into this problem.

Analytical forms of the wave functions for both $S$ and $D$ states for $n=0,1,2, \ldots$ for $c \bar{c}$, and $b \bar{b}$ systems thus obtained are given in Eq.(41). These wave functions were then used to calculate the decay constants and decay widths for various processes involving these quarkonia. We have also plotted the graphs of all the wave functions for the states, $1 S, \ldots, 4 S$ for $\eta_{c}$ and $\eta_{b}$ mesons, and for the states $1 S, 2 S, 1 D, 3 S, 2 D, 4 S$, and $3 D$ for $J / \Psi$ and $\Upsilon$ mesons, in Figs. 1-4. The over all features of all these plots show that the states $n S$ and $n D$ have $n-1$ nodes in their wave functions.

We are not aware of any other BSE framework, involving $4 \times 4$ BS amplitude, that treats the mass spectral problem involving heavy quarkonia analytically. To the best of our knowledge, all the other $4 \times 4$ BSE approaches treat this problem numerically just after they obtain the coupled set of Salpeter equations (see [18]). We further wish to mention that the over all features of our plots of wave functions in Eqs.(41) derived in our framework are very similar to the corresponding plots of wave functions obtained by purely numerical methods in [18], suggesting that our approach is not only in good agreement with the numerical approaches followed in other works, but also gives a deeper insight into the problem, by showing an explicit 
dependence of the mass spectrum on principal quantum number $N$ as in Eqs.(42-43). As mentioned above, we next intend to incorporate the OGE effects into the mass spectral equations perturbatively. However, this we intend to do as further work.

We also intend to extend this study to calculation of observables of heavy-light mesons such as $D$ and $B$, which would involve incorporation of unequal mass kinematics in our framework, which is beyond the scope of the present paper, and we wish to do as further work. This study will also be extended to processes involving quark-triangle diagrams with two or more hadronic vertices such as to decays $V \rightarrow P \gamma$, and $V \rightarrow P P$ (with $P$ and $V$ being the pseudoscalar and vector quarkonia respectively), as further works. It is further expected that our present framework (with $4 \times 4$ representation of two-body BS amplitude) will be able to overcome the complexities in the amplitudes for these processes that appeared in our earlier framework 42$]$.

Acknowledgements: This work was carried out at Addis Ababa University (AAU). We are thankful to the Physics Department, AAU for the facilities provided during the course of this work. HN thanks Samara University for support for his Doctoral programme. 


\section{References}

[1] E.E.Swanson, Phys. Rep.429, 243 (2006).

[2] N.Brambilla et al., Eur. Phys, J. C71, 1534 (2011).

[3] G. Bonvicini et al. (CLEO Collaboration), Phys. Rev. D 81, 031104 (2010).

[4] K. M. Ecklund et al. (CLEO Collaboration), Phys. Rev. D 78, 091501 (2008).

[5] B. Auger et al. (BABAR Collaboration), Phys. Rev. Lett. 103, 161801 (2009).

[6] C.McNielle, C.T.H.Davies, E.Follana, K.Hornbostel, G.P.Lepage (HPQCD Collaboration), arxiv: 1207.0994[hep-lat] (2012).

[7] G.S.Bali, K.Schiling, and A. Wachter, Phys Rev. D 56, 2566 (1997).

[8] T.Burch et al.(Fermilab and MILC Collaboration), Phys. Rev. D81, 034508 (2010).

[9] J.Gasser, H.Leutwyler, Ann. Phys. 158, 142 (1984).

[10] M.Neubert, Phys. Rep. 245, 259 (1994).

[11] M.A.Shifman, A.I.Vainshtein, V.I.Zakharov, Nucl. Phys. B147, 385, 448 (1979).

[12] E.Veli Veliev, K.Azizi, H.Sundu, N.Aksit, J.Phys. G39, 015002(2012).

[13] G.T.Bodwin, E.Braaten, G.P.Lepage, Phys. Rev. D51, 1125 (1995).

[14] C.H.L.Smith, Ann. Phys. (N.Y.) 53, 521 (1969).

[15] A. N. Mitra, B. M. Sodermark, Nucl. Phys. A695, 328 (2001).

[16] C. R. Munz, Nucl. Phys. A609, 364 (1996).

[17] R. Alkofer, L. W. Smekel, Phys. Rep.353, 281 (2001).

[18] C-H. Chang, G. L. Wang, Sci. China G53, 2005 (2010).

[19] R. Ricken, M. Koll, D. Merten, B. Metsch, H. Petry, Eur. Phys. J. A9, 221 (2000).

[20] A.N.Mitra, S.Bhatnagar, Intl. J. Mod. Phys. A07, 121 (1992).

[21] Bhagyesh, K.B.V.Kumar, A.P.Monterio, J.Phys. G38, 085001 (2011). 
[22] S.Godfrey, N. Isgur, Phys. Rev. D32, 189 (1985).

[23] T.Babutsidze, T.Kopaleishvili, A. Rusetsky, Phys. Rev. C59, 976 (1999).

[24] C.B.Yang, X.Cai, Phys. Rev. D51, 6332 (1995).

[25] M. R. Ahmady and R. R. Mendel, Phys. Rev. D 51, 141 (1995).

[26] D. Ebert, R. N. Faustov, and V. O. Galkin, Mod. Phys. Lett. A 18, 601 (2003).

[27] C. W. Hwang and Z. T. Wei, J. Phys. G 34, 687 (2007).

[28] H. W. Huang, J. H. Liu, J. Tang, and K. T. Chao, Phys. Rev. D 56, 368 (1997).

[29] C. S. Kim, T. Lee, and G. L. Wang, Phys. Lett. B 606, 323 (2005) arXiv:hep-ph/0411075.

[30] J. P. Lansberg and T. N. Pham, Phys. Rev. D 74, 034001 (2006); 75, 017501 (2007).

[31] J. J. Dudek and R. G. Edwards, Phys. Rev. Lett. 97, 172001 (2006)

[32] B.Auger et al.(BaBar collaboration), Phys. Rev. Lett. 103, 161801 (2009).

[33] K.F.Chen et al.(Belle collaboration), Phys. Rev. D82, 091106(R) (2010).

[34] K.W.Edwards et al.(CLEO collaboration), Phys. Rev.Lett. 86, 30 (2001).

[35] K.A.Olive et al., (Particle Data Group), Chin. Phys. C38, 090001 (2014).

[36] A. N. Mitra, Proc. Indian Natl. Sci. Acad. 65, 527 (1999).

[37] S.Bhatnagar, D.S.Kulshreshtha, A.N.Mitra, Physics Letters B 263, 485 (1991).

[38] S. Bhatnagar, S-Y. Li, J. Phys. G32, 949 (2006)

[39] S. Bhatnagar, S-Y.Li, J. Mahecha, Int. J. Mod. Phys. E20, 1437 (2011).

[40] S.Bhatnagar, J.Mahecha, Y.Mengesha, Phys. Rev. D90, 014034 (2014).

[41] J.T.Laverty, S.F.Radford, W.W.Repko (2009) [arXiv:hep-ph/0901.3917].

[42] E.Mengesha, S.Bhatnagar, Intl. J. Mod. Phys. E22, 1350046 (2013).

[43] H.Negash and S.Bhatnagar, Intl. J. Mod. Phys. E24, 1550030 (2015)

[44] H. J. Munczek and P. Jain, Phys. Rev. D 46, 438 (1992); P. Jain and H. J. Munczek, Phys. Rev. D 48, 5403 (1993). 
[45] V.Sauli, Phys. Rev. D86, 096004 (2012). .

[46] M. G. Olsson, A. D. Martin, and A.W. Peacock, Phys. Rev. D 31,81 (1985).

[47] S. Chakravorty, K. K. Gupta, N. N. Singh, and A. N. Mitra, Prog. Part. Nucl. Phys. 22, 43 (1989).

[48] T.Peng, B-Q.Ma, Eur. Phys. J. A48, 66 (2012), arxiv: 1204.0863[hep-ph].

[49] D.Ebert, R.N.Faustov, V.O.Galkin, Phys. Atomic Nuclei 76, 1554 (2013); arXv:1111 .0454v1[hep-ph] (2011).

[50] J.J.Dudek, R.G.Edwards, D.G.Richards (JLab Lattice group), Phys. Rev. D73, 074507 (2006); hep$\mathrm{ph} / 0601137$.

[51] T.Kawanai, S.Sasaki, arXiv:1503.05752[hep-lat]

[52] G-L.Wang, Phys. Lett. B633, (2006).

[53] S.Bhatnagar,M.Li, S-Y.Li, J. Mod. Phys. 5, 1027 (2014). 